\title{
Genesis and Development of Spring Rainstorms in Northern Southeast Asia: Southwest China-Northern Indochina and the Northern South China Sea
}

\author{
TSING-CHANG CHEN AND JENQ-DAR TSAY \\ Department of Geological and Atmospheric Sciences, Iowa State University, Ames, Iowa \\ MiNG-CHANG YeN \\ Department of Atmospheric Sciences, National Central University, Chung-Li, Taiwan
}

(Manuscript received 9 March 2017, in final form 21 October 2017)

\begin{abstract}
During May and June, the monsoon rainfall in northern Southeast Asia is primarily produced by rainstorms. At the mature stage, these storms, coupled with a midtropospheric subsynoptic-scale trough, produce rainfall $\geq 50 \mathrm{~mm}(6 \mathrm{~h})^{-1}$ and exhibit a cyclonic surface vortex. With a scale $\sim O\left(10^{2}\right) \mathrm{km}$, rainstorms during the period of 1979-2016 are identified with station and satellite observations, along with assimilation data. Several dynamic processes of rainstorm geneses are disclosed by an extensive analysis. 1) Maximum occurrence of rainstorm geneses is located in the midtroposphere of two regions (northern Vietnam-southwestern China and the northern South China Sea), but eventually penetrates downward to the surface. 2) The environment favorable for rainstorm genesis is a southwest-northeast-oriented narrow trough formed by the confluence of the midtropospheric northeasterly around the eastern Tibetan Plateau and the lower-tropospheric monsoon southwesterlies. Because the criterion for Charney-Stern instability is met by the shear flow of this narrow trough, rainstorms are likely initiated by this instability. 3) The majority of rainstorm geneses occurs during the evening over the land and the morning at sea. This timing preference is caused by the modulation of the clockwise rotation of the East Asia continent circulation in response to the diurnal variation of the land-sea thermal contrast. These new findings from this study offer not only a new perspective for the genesis mechanism of the late spring-early summer rainstorms in northern Southeast Asia, but also a new initiative to develop medium-range forecasts for these rainstorms.
\end{abstract}

\section{Introduction}

The warm-season rainfall variation over south China and Taiwan undergoes an active rainfall period (May and June) and a typhoon (TY) active period (mid-July to early October) separated by a midsummer (late Juneearly July) dry spell (Ramage 1952), as illustrated by Fig. 1a. During the active monsoon phase, the frontal activity diminishes, but the monsoon southwesterly flow intensifies. Some midtropospheric disturbances accompanied by heavy rainfall moving along this monsoon southwesterly flow may produce heavy precipitation

\footnotetext{
Supplemental information related to this paper is available at the Journals Online website: https://doi.org/10.1175/MWR-D-170059.s1.
}

Corresponding author: Tsing-Chang (Mike) Chen, tmchen@ iastate.edu over south China and southwest Taiwan. Caused by the northward migration of the western subtropical anticyclone, rainfall over south China and Taiwan significantly decline over the break monsoon phase. This northward advance of the subtropical anticyclone is followed by the intertropical convergence zone (ITCZ), the increase of TY occurrence frequency over the western tropical $\mathrm{Pa}$ cific, and rainfall enhancement to reach its second maximum in late July.

Ramage's depiction of the warm-season rainfall variation for south China and Taiwan was adopted by the East Asian monsoon community to portray the East Asian monsoon life cycle; mei-yu-baiyu-break-typhoon season (e.g., Chang 1972; Lau and Li 1984; Ninomiya and Murakami 1987). Using multidata sources, this monsoon life cycle around the East China Sea and the northern part of the South China Sea (SCS), including southern China, Taiwan, Japan, and South Korea, was comprehensively analyzed by Chen et al. (2004). Despite 
(a) East/Southeast Asian monsoon life cycle: Taiwan rainfall

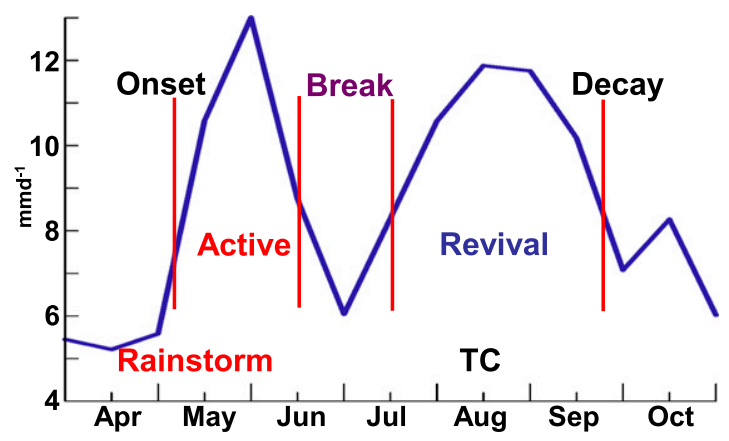

(b) Rainfall contributed by different weather systems

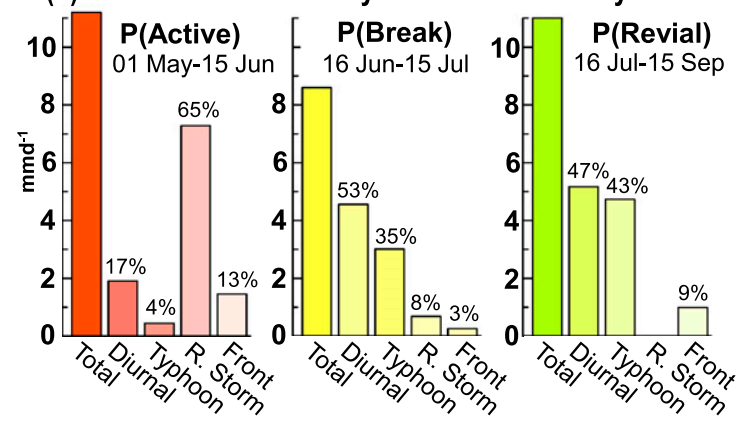

FIG. 1. (a) Life cycle of the Southeast/East Asia summer monsoon [active phase (1 May-15 Jun), break phase (15 Jun-15 Jul), and revival phase (16 Jul-15 Sep)], depicted with the climatological 15-day-mean rainfall for Taiwan from April to October (adopted from Chen et al. 2004) and (b) the rainfall in Taiwan produced by different weather systems (including diurnal variations, typhoons, rainstorms, and fronts) for different phases of the summer monsoon life cycle (adopted from Chen et al. 2011).

this well accepted depiction of the East Asian warmseason monsoon life cycle, the IR image from the Geostationary Meteorological Satellite (GMS) for the eastward-propagating rainstorms in the northern SCS (Fig. 2) during the SCS Monsoon Experiment (SCSMEX; Lau 1997; Lau and Yang 1997) leads us to infer their crucial role in the rainfall contribution during the active monsoon phase in the southern part of East Asia. This inference was supported by our pilot studies (Fig. 1b; Chen et al. 2004; Wang and Chen 2008; Chen et al. 2011).

To understand the rain-producing process during the active monsoon phase over southwest Taiwan, the Southwest Monsoon Experiment/Terrain-Influenced Monsoon Rainfall Experiment (SoWMEX/TiMREX) (Jou et al. 2011) was conducted over May and June for 2008. This active monsoon phase was separated into two periods (Davis and Lee 2012). Major rainfall during the first period (15 May-2 June) was primarily produced by afternoon convection over the elevated terrain of southwest Taiwan. The second period (3-30 June) covered three rain spells; each lasted about 5-6 days over either the southwest plains or the elevated region $(\geq 200 \mathrm{~m})$. The postexperiment analyses focused on two spells (1-6 June) and (12-17 June). During the former spell, a series of three MCSs occurred south of a front moving across southern Taiwan: two rainfall events (3 and 4 June) followed by a mesoscale convective vortex (MCV; 5 June) on the southwestern coast plain of Taiwan (Lai et al. 2011). The latter spell included two heavy rainfall events. The first event exhibited an eastward propagation of MCS, while the second event showed a westward propagation of MCS (Wang et al. 2014). These efforts shed light on the synoptic conditions and physical processes in developing these heavy rainfall events over southwestern Taiwan during the active summer monsoon phase.

Despite efforts made by previous studies (Davis and Lee 2012; Lai et al. 2011; Wang et al. 2014), the genesis/ formation mechanism of MCSs or MCV observed by these studies during the active summer monsoon phase is still beyond reach. The rainfall produced by these MCSs often leads to disastrous floods over some monsoon regions. To manage the water supply, reduce traffic hazards, and mitigate disasters caused by these heavy rainfall events, forecast improvement of these events is necessary. A better understanding of the genesis and development mechanisms facilitates our pursuit of this goal. It is inferred from the IR image of MCSs (Fig. 2) that the geneses of these rainfall events occur in the upstream region of monsoon westerlies during the active monsoon phase. This inference is confirmed by tracking rainfall at three locations along the monsoon westerlies [Hanoi, Vietnam (WMO 48820), Hong Kong (WMO 45004), and southwest Taiwan] during 15 May20 June 2008 [Late Spring-Early Summer Rainstorm Experiment (LSESREX), Taiwan EAMEX Committee (2007)]. As inferred from Fig. 3, geneses of these rainfall events may occur over the southwestern Chinanorthern Indochina region and the northern SCS. There is no formal definition for the heavy rain events during the active monsoon phase of East Asia, but they are commonly named "rainstorm" when the local rainfall

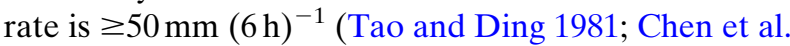
1998). This term is adopted by this study. An extensive analysis for rainstorm formation during the active summer monsoon phase is reported in this study. Our efforts include a backtracking procedure, genesis mechanism, and diurnal variation in the genesis over land and sea.

To achieve these goals, this manuscript is organized in the following manner. Section 2 describes an identification of downstream development and the backtracking procedure for rainstorms to their genesis 


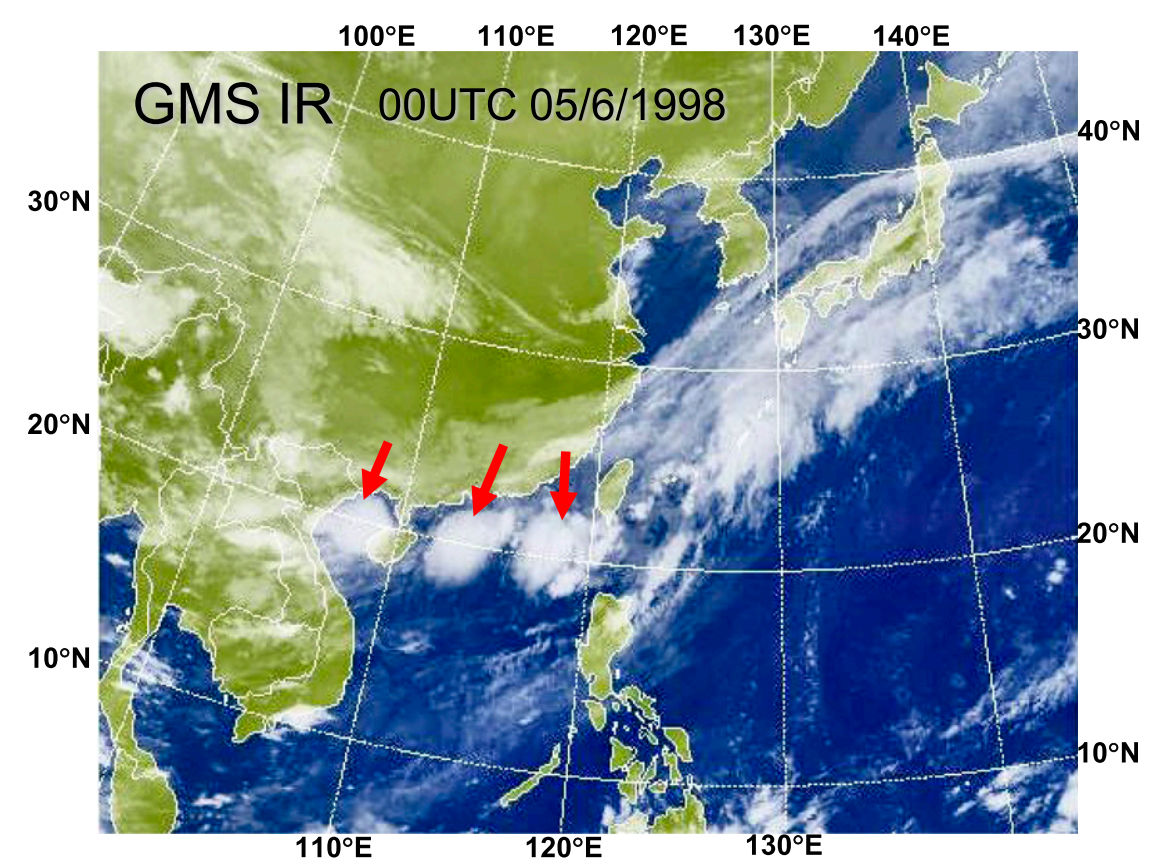

FIG. 2. The IR image for a series of MCSs/rainstorms between the Gulf of Tonkin and Taiwan at 0000 UTC 5 Jun 1998 during the South China Sea Monsoon Experiment (SCSMEX).

locations. Data utilized for this analysis are also presented. Section 3 documents the occurrence statistics and geographic/spatial distribution of rainstorm genesis for the 1979-2016 period. After monsoon onset in the South China Sea during mid-May, the low-level monsoon westerlies intensify across Indochina. Following the development of the Tibetan anticyclone, the anticyclonic flow develops around the Tibetan Plateau. The confluence of these two monsoon flows forms a positive shear flow extending from the Yangtze River valley to southwest China, a favorable environment for rainstorm genesis. A detailed synoptic analysis related to the rainstorm genesis both in the southwestern China-northern Vietnam region and the northern South China Sea is presented in section 4. Because the Tibetan anticyclone exists in the upper troposphere, rainstorm genesis occurs similar to the African easterly waves south of the African easterly jet in the midtroposphere (e.g., Burpee 1972). For this reason, the Charney-Stern instability criterion (Charney and Stern 1962) is applied to explore the possible genesis mechanism of the rainstorm. In response to the land-sea thermal contrast, the diurnal variation of the atmospheric circulation undergoes a clockwise rotation over the East Asian continent and adjacent oceans (Chen 2006). Because the rainstorm genesis exhibits a land-sea diurnal variation, the possible impacts on the diurnal variation of rainstorm genesis by diabatic heating and the regional circulation are illustrated in section 5. Finally, section 6 presents a summary of new findings from this study. Future studies about rainstorm impacts in monsoon development, an intraseasonal mode on the rainstorm activity, and possible development of East Asian rainstorms from the eastward-propagating midtropospheric cyclones in South Asia are suggested.

\section{Identification of rainstorm and analysis data}

It has been perceived by the East Asian meteorological community that rainstorms during the active monsoon phase develop from MCSs associated with the mei-yu-baiu front (e.g., Lau and Li 1984; Ninomiya and Murakami 1987). Research has focused on the structure, development, and weather/rainfall impacts from these rainstorms, but rarely on the genesis mechanism and large-scale environment favorable for genesis. To explore the latter two issues, rainstorms should be correctly identified and backtracked to their genesis locations. Because of a lack of understanding in its genesis mechanism, rainstorms are easier to identify during their mature phase than their genesis phase. To avoid any possible error in search of genesis locations, rainstorms should be properly identified so a backtracking procedure can be performed. Some insight on the genesis mechanism can then be revealed from locations and the associated synoptic conditions. 


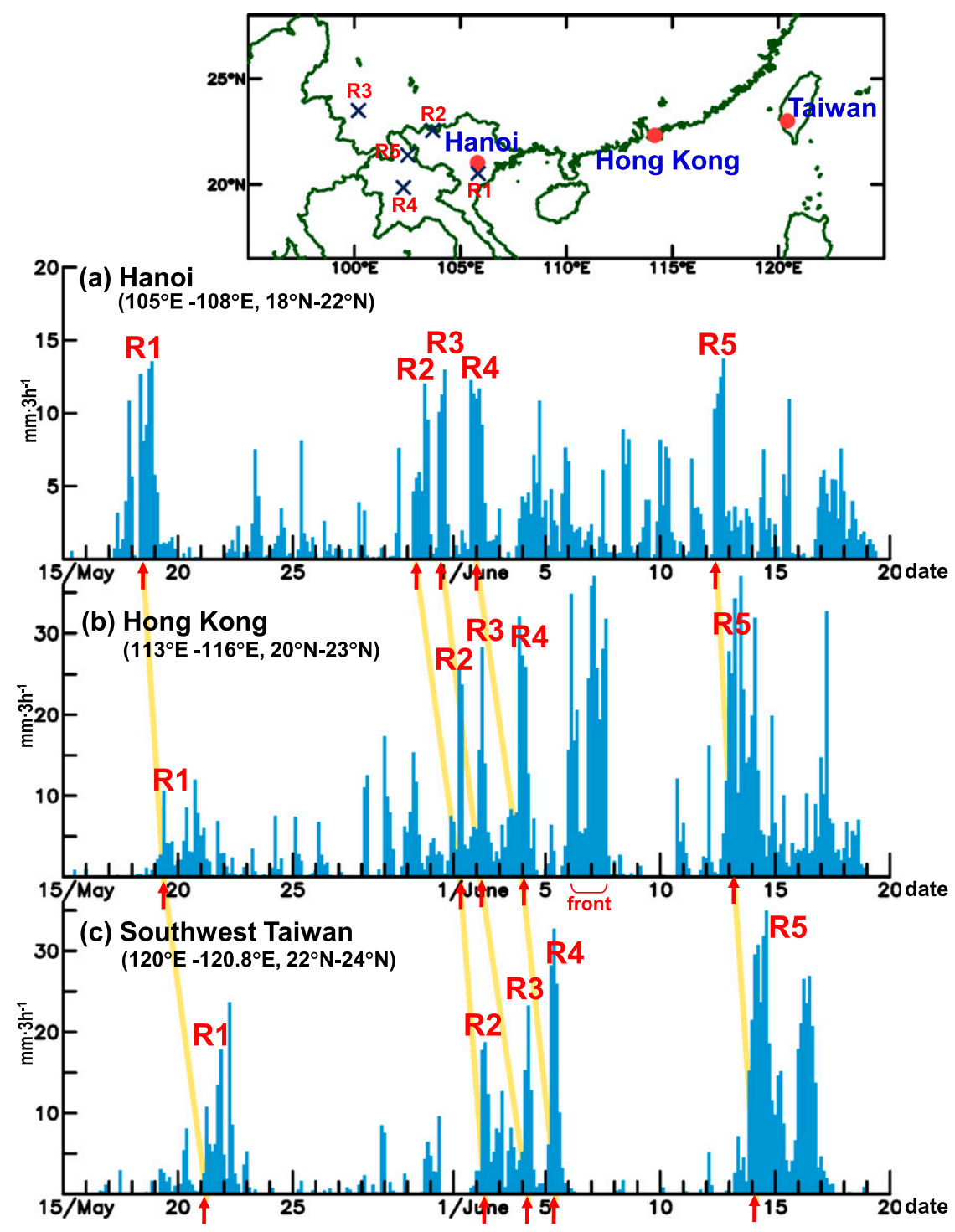

FIG. 3. Rainfall [mm $(3 \mathrm{~h})^{-1}$ ] histograms for 15 May-20 Jun 2008: (a) Hanoi, (b) Hong Kong, and (c) southwest Taiwan $\left(22^{\circ}-24^{\circ} \mathrm{N}, 120^{\circ}-120.6^{\circ} \mathrm{E}\right)$. Dates of five identified rainstorms at these three locations are marked with red arrows along the time axis and by $\mathrm{RN}(N=1, \ldots, 5)$ on the rainfall histograms. These three locations are marked with red dots on the top geographical panel including genesis locations of five rainstorms (marked by blue crosses).

\section{a. Identification criteria for a rainstorm}

A typical rainstorm shown in Fig. 4 is utilized to illustrate the criteria used herein:

1) A rainstorm reaches a rainfall amount $\geq 50 \mathrm{~mm}$ $(6 \mathrm{~h})^{-1}$ at the mature stage of its life cycle (Chen et al. 1998).

2) Relative vorticity of a rainstorm at its genesis location in the midtroposphere (700-500-hPa layer) should reach a threshold value of $\geq 2 \times 10^{-5} \mathrm{~s}^{-1}$. This vorticity value becomes larger and penetrates downward, when a surface vortex appears at some later stage.

3) A rainstorm (indicated by a red oval in Fig. 4c) is coupled with a midtropospheric subsynoptic-scale trough (a thick short red line) (Fig. 4b). A cold, surgelike flow (blue arrow) and a narrow trough (red-dashed line) are located behind an uppertropospheric short-wave trough (Fig. 4c).

4) Underneath the midtropospheric subsynoptic-scale trough, the rainstorm, a cyclonic vortex in the lower troposphere, is identified either by a surface 

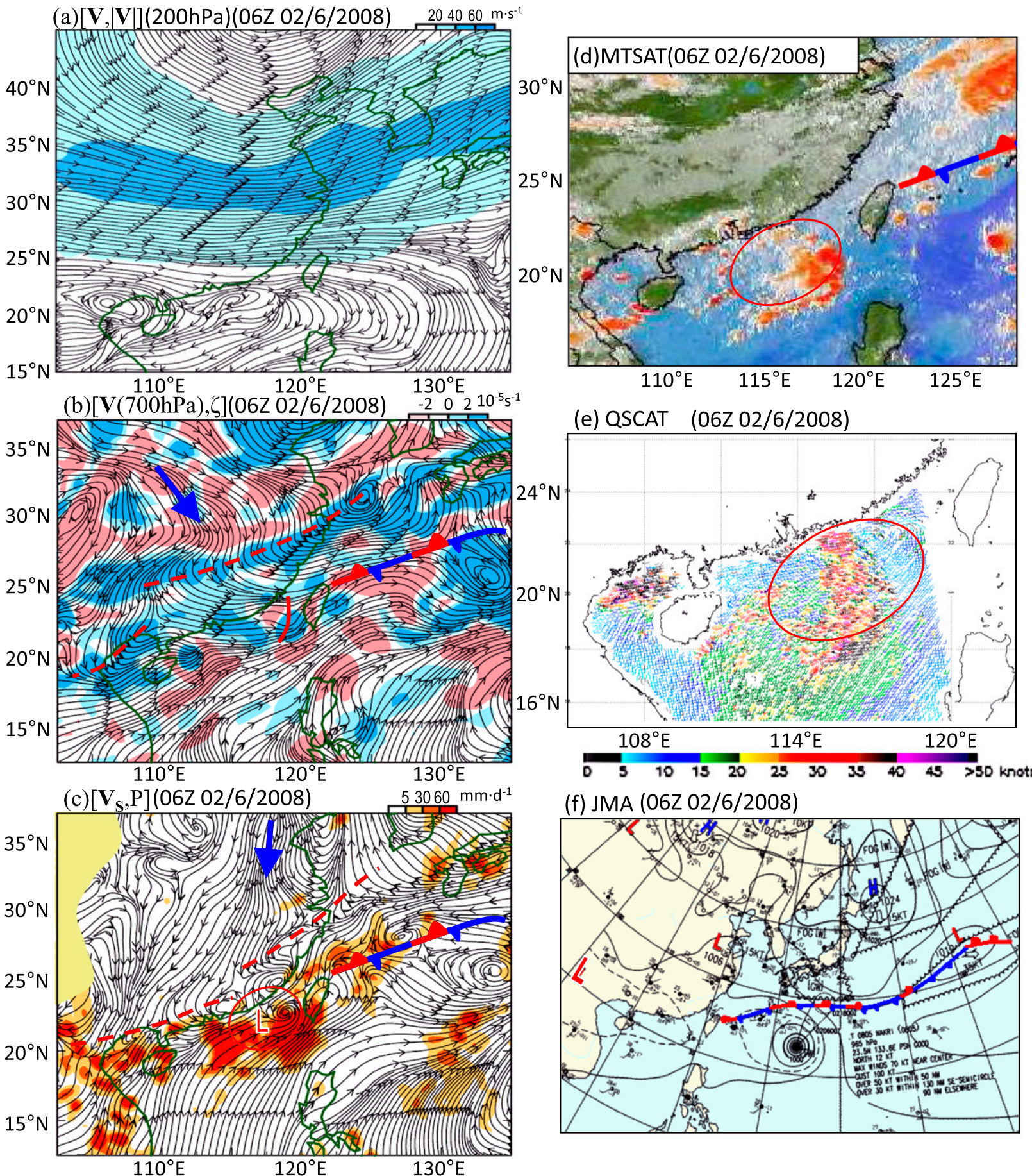

(f) JMA (06Z 02/6/2008)

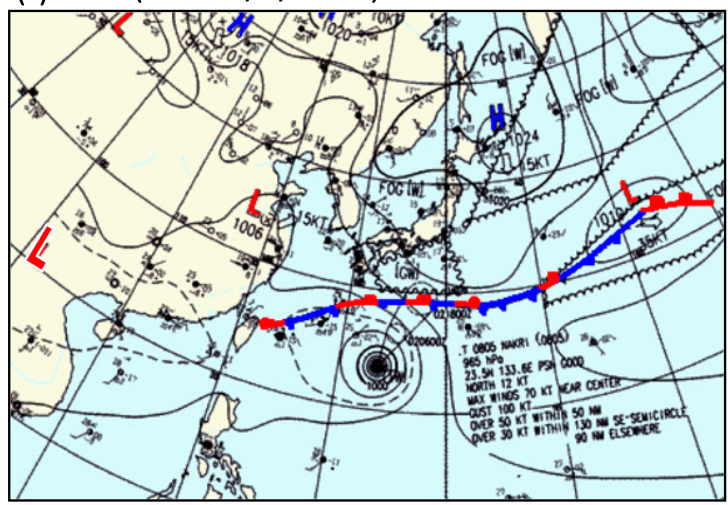

FIG. 4. A typical rainstorm case identified at 0600 UTC 2 Jun 2008: (a) 200-hPa streamline chart with isotachs (blue). (b) 700-hPa streamline chart superimposed with vorticity (red-blue), a rainstorm trough line (short red solid line), a shear line (red-dashed line), a front, and a cold surgelike flow (dark blue arrow). (c) Surface streamline chart superimposed with TRMM rainfall (gold), a shearline (red-dashed line), a front, a cold surgelike flow (dark blue arrow), and the low pressure system marked by a red L. (d) IR image of MTSAT. (e) Surface wind vectors of QuikSCAT. (f) JMA surface analysis chart. Scales for isotachs, vorticity, and TRMM rainfall are shown on the top-right-hand panels of (a)-(c), respectively. The rainstorm is marked with a red oval in (c)-(e). 
(a) IR image of MTSAT
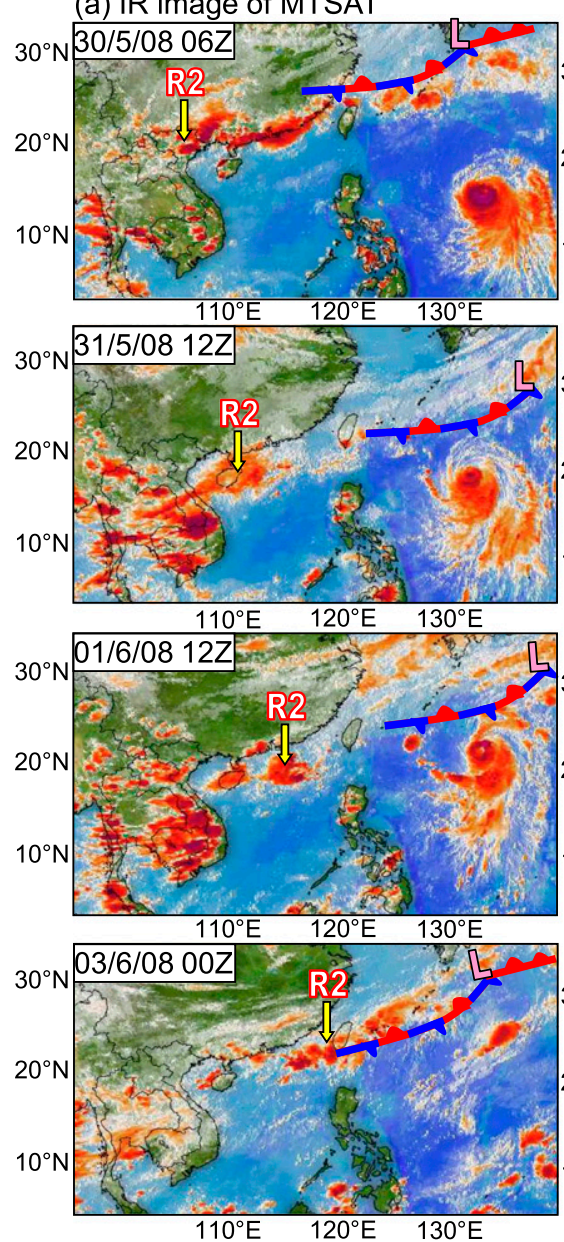

(b)[V(600hPa),P]
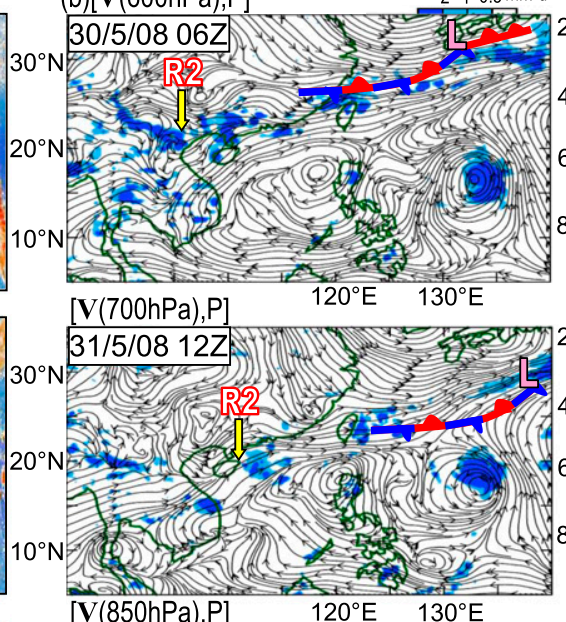

(c) Longitude-height cross-section of $\left(\zeta, \zeta_{\mathrm{t}}\right)$
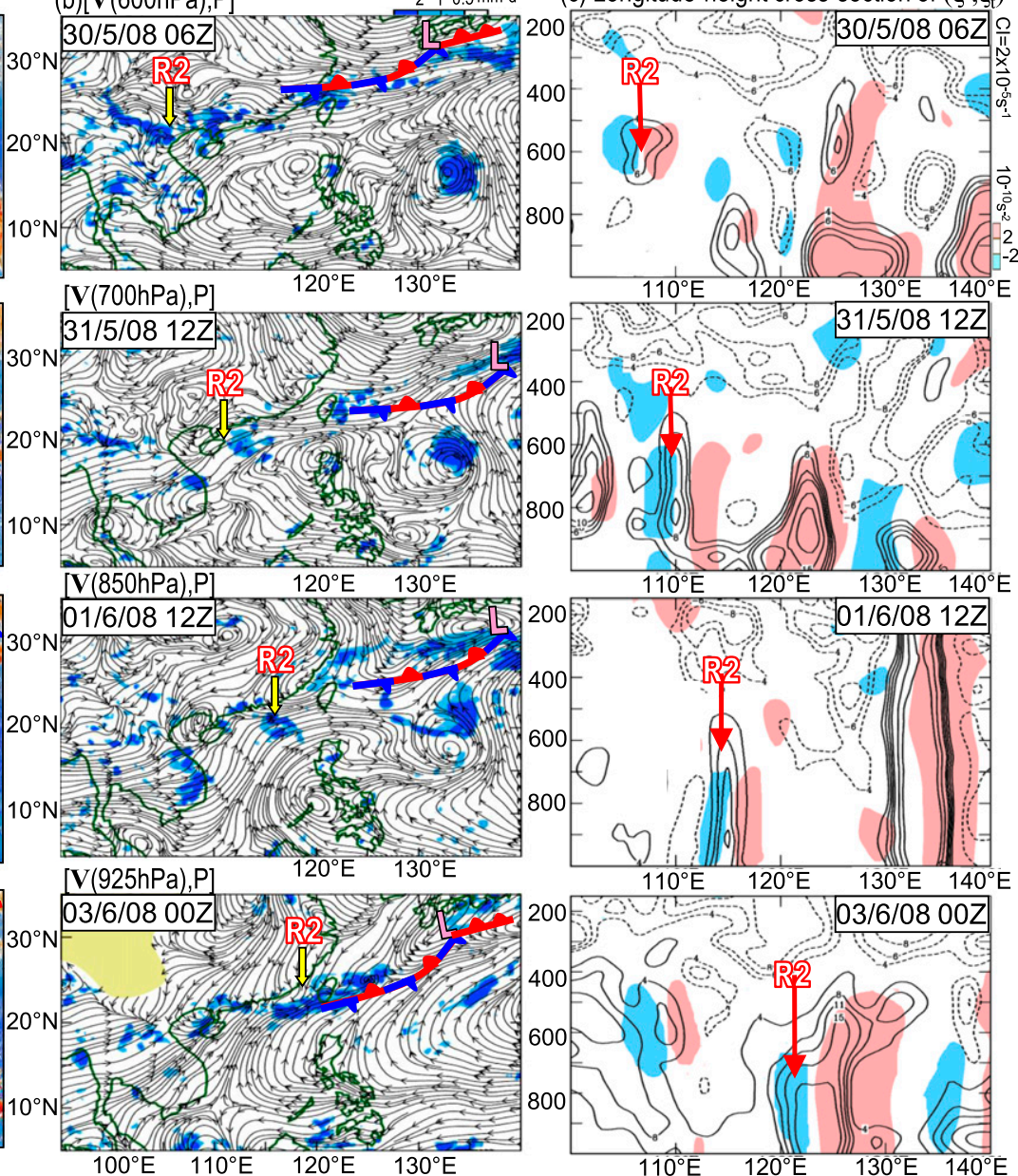

FIG. 5. Temporal evolution of the second rainstorm identified in the summer of 2008 from its genesis at 0800 UTC 30 May-0000 UTC 3 Jun 2008 (when a great flood occurred in southwestern Taiwan): (a) IR image of MTSAT superimposed with front, (b) 925-hPa streamline chart superimposed with TRMM rainfall (blue) and front, (c) longitude-height cross section of $\left(\zeta, \zeta_{t}\right)\left(\zeta\right.$ and $\zeta_{t}$ are vorticity and vorticity tendency, respectively) cutting through the latitude of the rainstorm, and $x-t$ diagrams of (d) TRMM rainfall $P$, (e) $\zeta(600 \mathrm{hPa})$, and (f) $\zeta(850 \mathrm{hPa})$ over the period $15 \mathrm{May}-19 \mathrm{Jun} 2008$. Genesis location of rainstorm R1 is marked with a blue cross on an orographic map of Southeast Asia on the top panels of (d)-(f). The location of the rainstorm is marked with R2 in (a)-(c) and all five identified rainstorms in May-June 2008 are marked with R1-R5 in (d)-(f). In (c), the contour interval of $\zeta$ is $2 \times 10^{-5} \mathrm{~s}^{-1}$ and $\zeta_{t}>2 \times$ $10^{-10} \mathrm{~s}^{-2}\left(<-2 \times 10^{-10} \mathrm{~s}^{-2}\right)$ shaded by red (blue). Trajectories of five rainstorms R1-R5 are indicated by blue dot lines in the three $x-t$ diagrams (bottom row) of (d)-(f).

streamline chart (Fig. 4c) or by QuickSCAT surface winds (Fig. 4e), if available.

5) Some MCS may be embedded/merged with a front, but the rainstorm in its initial stage is often located west of a JMA-analyzed front's tail (Fig. 4f).

6) Despite its genesis location, the trajectory of a rainstorm should reach the vicinity or east of Taiwan $\left(\sim 120^{\circ} \mathrm{E}\right)$ (Fig. 5a). The life cycle for this storm should be $\geq 2$ days.

7) Measured by the size of the cyclonic vortex at the surface (Fig. 4e), the subsynoptic-scale disturbance in the midtroposphere (Fig. 4b), or the IR image of the Multifunction Transport Satellite (MTSAT)
(Fig. 4d), the horizontal scale for a rainstorm is smaller than a synoptic-scale disturbance $\left[\sim O\left(10^{3}\right) \mathrm{km}\right]$, but larger than an $\operatorname{MCS}\left[\sim O\left(10^{2}\right) \mathrm{km}\right]$.

\section{b. Backtracking procedure to determine genesis location of rainstorm}

Rainstorms, recognizable weather disturbances, are not difficult to identify, except during their genesis stage. Several diagnostic tools with different data sources are applied to backtrack these storms to their initial locations. The first group of diagnostics consists of IR images for GMS/MTSAT (Fig. 5a), surface/925-hPa streamline 
(d) $\mathrm{P}$
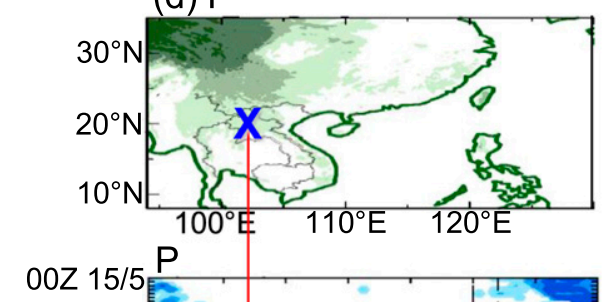

(e) $\zeta(600 \mathrm{hPa})$

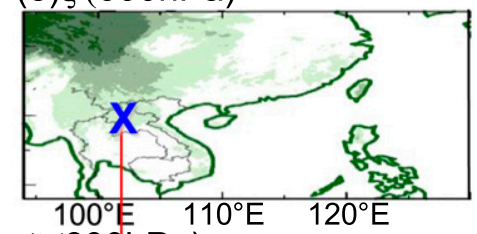

$\zeta(600 \mathrm{hPa})$

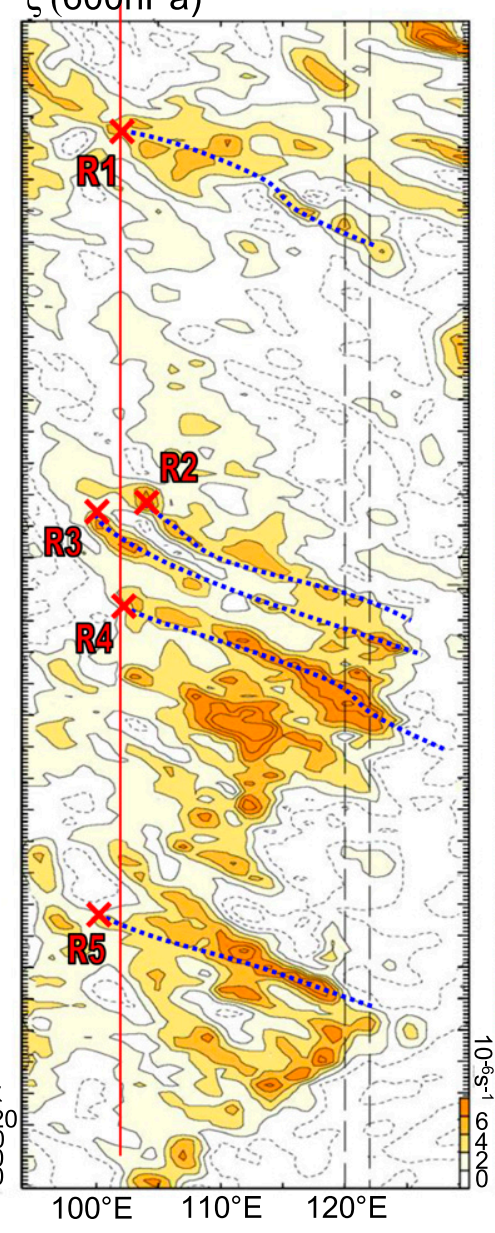

(f) $\zeta(850 \mathrm{hPa})$

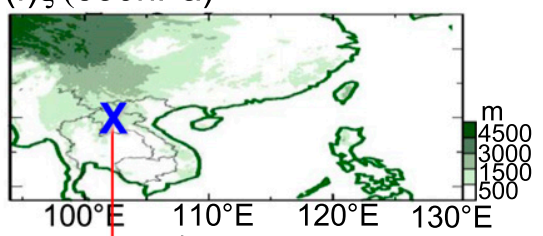

$\zeta(850 \mathrm{hPa})$

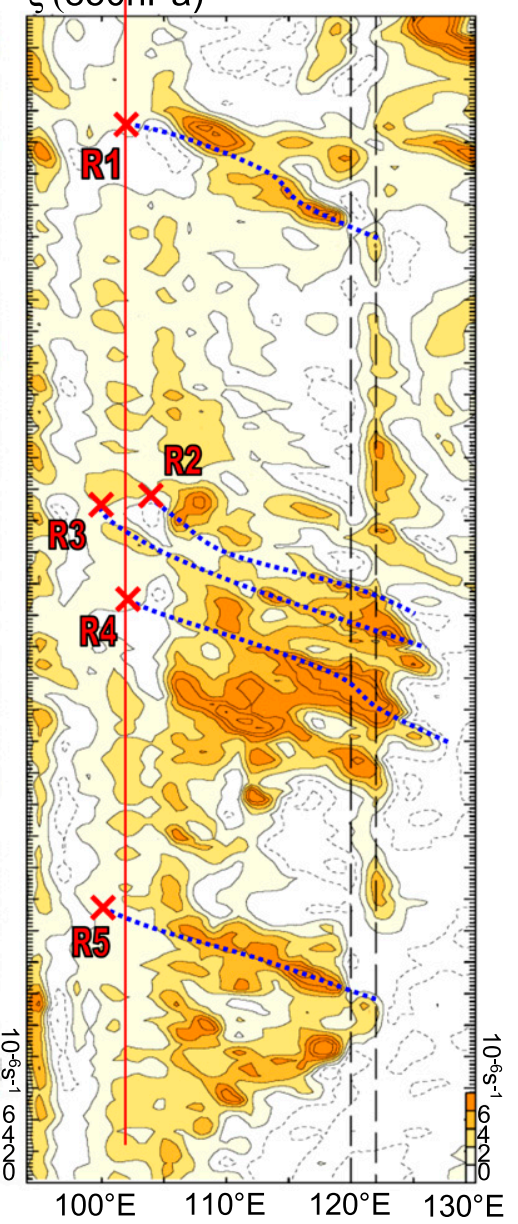

FIG. 5. (Continued)

charts along with precipitation (TRMM or GPCP; Fig. 5b), and the longitude-height cross sections of vorticity, which cuts through the genesis and propagation paths of the rainstorm (Fig. 5c). The second group of diagnostic tools are the longitude-time diagrams of rainfall (TRMM/ GPCP; Fig. 5d) along the trajectory and vorticity at $600 \mathrm{hPa}$ [ $\zeta(600 \mathrm{hPa}) ;$ Fig. $5 \mathrm{e}]$ and vorticity at $850 \mathrm{hPa}[\zeta(850 \mathrm{hPa})$; Fig. $5 \mathrm{f}]$ along the identified path of the rainstorms.

As arranged in Fig. 5, horizontal charts provide a clear view of the temporal-spatial evolution of a rainstorm. The spatial relationship between the rainstorm genesis location and Asian front on JMA surface maps provide a perspective different from the conventional view that rainstorms evolve from MCSs embedded in a mei-yu front. The synoptic conditions of a rainstorm's genesis location and its downstream development may be depicted by streamline charts prepared with the NCEP GFS and CFS reanalyses. The QuikSCAT observation is formed with passes over the analyzed region twice daily. Thus, the existence of a rainstorm vortex may be confirmed with QuikSCAT surface wind vectors over the sea. Rainstorm genesis occurs in the midtroposphere $(700-500 \mathrm{hPa})$. Therefore, the downward penetration of the rainstorm can be illustrated with longitude-height cross sections of vorticity and vorticity tendency. The propagation properties and downstream development of a rainstorm can be revealed from the longitude-time diagrams for precipitation $P, \zeta(600 \mathrm{hPa})$, and $\zeta(850 \mathrm{hPa})$ 
TABLE 1. Dataset-I data: Surface and upper-air wind fields.

\begin{tabular}{|c|c|c|c|c|c|}
\hline Dataset & Source & Spatial resolution & Temporal resolution & Data period & Source information \\
\hline \multirow[t]{3}{*}{$\begin{array}{l}\text { Reanalyses and Global } \\
\text { Forecast System }\end{array}$} & $\begin{array}{l}\text { NASA } \\
\text { MERRA }\end{array}$ & $1.25^{\circ}($ lon $) \times 1.25^{\circ}$ (lat) & $3 \mathrm{~h}$ & 1979-present & Rienecker et al. (2008) \\
\hline & NCEP GFS & $0.5^{\circ}$ lon $\times 0.5^{\circ}$ lat & $6 \mathrm{~h}$ & 2006-present & $\begin{array}{l}\text { Global Climate and } \\
\text { Weather Modeling Branch, } \\
\text { EMC, NCEP }(2003,2016)\end{array}$ \\
\hline & NCEP CFSR & $0.5^{\circ}($ lon $) \times 0.5^{\circ}($ lat $)$ & 6 & 1979-2011 & Saha et al. (2010) \\
\hline Surface observation & QuikSCAT & $\begin{aligned} 12 \mathrm{~km} & \times 12 \mathrm{~km} \\
0.5^{\circ}(\text { lon }) & \times 0.5^{\circ}(\text { lat })\end{aligned}$ & Twice daily & 1999-2009 & Atlas et al. (2001) \\
\hline
\end{tabular}

with 6-h intervals. The genesis location for rainstorm $\mathrm{R} 2$ is also verified by the contrast between the longitude-time diagrams for $\zeta(600 \mathrm{hPa})$ and $\zeta(850 \mathrm{hPa})$ on the upstream side, since a $\zeta(600 \mathrm{hPa})$ center appears in Fig. $5 \mathrm{e}$, as indicated by Fig. $5 c$, but does not have a corresponding $\zeta(850 \mathrm{hPa})$ center in Fig. $5 \mathrm{f}$.

In addition to information revealed from Fig. 5, the following criteria are adopted to accurately identify the genesis location and time of rainstorm:

1) Following criterion 2 for the rainstorm identification in section $2 \mathrm{a}$ to track its genesis location, the embryo vortex should have maximum vorticity $\left(\zeta_{\max }\right) \geq 2 \times$ $10^{-5} \mathrm{~s}^{-1}$ and maximum $\zeta_{t}$ (maximum vorticity tendency) $\geq 1 \times 10^{-10} \mathrm{~s}^{-2}$, and $P \geq 0 \mathrm{~mm}$ day $^{-1}$ over a box of $5^{\circ} \times 5^{\circ}$ around the genesis location.

2) Synoptically, the rainstorm genesis is coupled with a midtropospheric subsynoptic-scale trough.

\section{c. Data}

A rainstorm is identified using criteria 2-6 with surface and upper-air winds, but assessed by all criteria including surface (WMO/GHCN) station and satellite (e.g., TRMM) rainfall measurements, global precipitation analyses (e.g., PERSIANN, GPCP), and IR satellite images or TBB. Based on these identification criteria for rainstorms, the data sources utilized for the first group of criteria are designated as dataset I (Table 1) and the second group of criteria as dataset II (Table 2). Because the analysis period is close to four decades, it is difficult (if not impossible) to process all data sources in a uniform manner. In addition, the identification and depiction of a rainstorm need the horizontal resolution of data to be $\leq O\left(10^{2}\right) \mathrm{km}$. Proper use of all available data sources for the above manual identification criteria of rainstorms over three decades is a time-consuming task.

\section{Statistics of rainstorm genesis}

Using the identification criteria for rainstorms and the backtracking procedure to locate their genesis positions as outlined in section 2, a total of 297 rainstorms and their genesis locations are identified during the period 1979-2016 (Table 3): 248 geneses occurred over the southwestern China-northern Vietnam region and the northern South China Sea, and 49 geneses over the Bay

TABLE 2. Dataset-II precipitation: Station rainfall, analyzed global precipitation, and rainfall proxy derived from satellite observations.

\begin{tabular}{|c|c|c|c|c|c|}
\hline Dataset & Source & $\begin{array}{l}\text { Spatial } \\
\text { resolution }\end{array}$ & $\begin{array}{l}\text { Temporal } \\
\text { resolution }\end{array}$ & Data period & Source information \\
\hline Station rainfall & WMO surface stations & $\begin{array}{l}\text { Over the region of } \\
\text { interest, particularly } \\
\text { Hong Kong and SW } \\
\text { Taiwan, Hanoi } \\
\text { (WMO 48820) }\end{array}$ & $\begin{array}{l}\text { Hourly/3-h } \\
\text { rainfall }\end{array}$ & 1979-present & \\
\hline \multirow{3}{*}{$\begin{array}{c}\text { Analyzed global } \\
\text { precipitation }\end{array}$} & TRMM (3B42v7) & $0.25^{\circ}($ lon $) \times 0.25^{\circ}$ (lat) & $3 \mathrm{~h}$ & $1998-2016$ & Huffman and Bolvin (2015) \\
\hline & PERSIANN & $0.25^{\circ}($ lon $) \times 0.25^{\circ}$ (lat) & Daily & 1983-present & Ashouri et al. (2015) \\
\hline & GPCP v2.2 & $2.5^{\circ}($ lon $) \times 2.5^{\circ}($ lat $)$ & Daily & 1979-present & Huffman and Bolvin (2013) \\
\hline \multirow{5}{*}{$\begin{array}{l}\text { Rainfall proxy } \\
\text { (IR image) }\end{array}$} & OLR & $2.5^{\circ}($ lon $) \times 2.5^{\circ}$ (lat) & Daily & 1974/2006-present & Liebmann and Smith (1996) \\
\hline & $\begin{array}{l}\text { TBB (bright temperature } \\
\text { of blackbody) }\end{array}$ & $1^{\circ}($ lon $) \times 1^{\circ}($ lat $)$ & $3 \mathrm{~h}$ & 1980-present & \\
\hline & $G M S-1-5$ & $5 \mathrm{~km} \times 5 \mathrm{~km}$ & $1 \mathrm{~h}$ & Jul 1977-May 2003 & $\begin{array}{c}\text { Blersch and Probert (1991); } \\
\text { Meteorological Services } \\
\text { Centre Japan (1997) }\end{array}$ \\
\hline & GOES-9 & $4 \mathrm{~km} \times 4 \mathrm{~km}$ & $1 \mathrm{~h}$ & May 1995-Feb 2005 & Clark (1983) \\
\hline & $M T S A T-1-2$ & $4 \mathrm{~km} \times 4 \mathrm{~km}$ & $1 \mathrm{~h}$ & Feb 2005-present & Faller (2005) \\
\hline
\end{tabular}




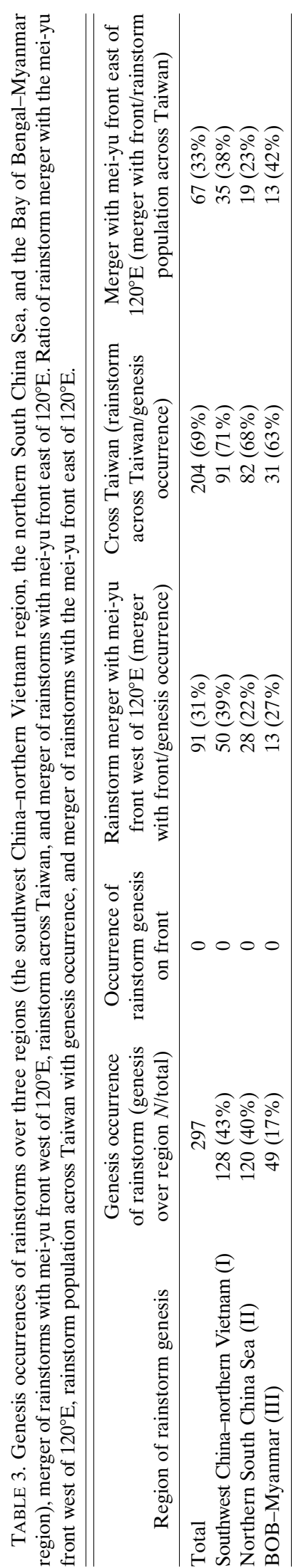

of Bengal (BOB)-Myanmar region. Two characteristics of rainstorm genesis in the latter region are different from those over the former region:

(i) The rainstorm genesis occurrence ahead of the midtropospheric BOB trough is overlaid by the upper-level Tibetan anticyclone.

(ii) To reach East Asia, the BOB-Myanmar rainstorms are propagated eastward by monsoon westerlies over a distance $\geq 2000 \mathrm{~km}$ through different weather systems across South Asia and northern Indochina.

For these reasons, the impact of the $\mathrm{BOB}$ trough on the genesis of the BOB-Myanmar rainstorms and the effects of different weather systems on the eastward propagation of these rainstorms will be reported in a separated study. The present study focuses only on the 248 rainstorm geneses. Salient features for these genesis locations are characterized as follows.

\section{a. Geographic location of genesis}

Genesis locations and trajectories of identified rainstorms are shown in Figs. 6a and 6b. Interestingly, the timing of the genesis is generally divided into two groups: afternoon-evening (0600-1200 UTC, red dots) and midnight-morning (1800-0000 UTC, blue dots). Most of the former group appear over land, while most of the latter group occur over the sea. This land-sea contrast of genesis timing should be modulated by the large-scale environmental flow through its response to the diurnal variation in radiative heating. The population of genesis $\left(N_{F}\right)$ (Fig. 6c) exhibits triple maxima along the longitudinal direction. A major maximum, $N_{F}$, appears over land, particularly along the eastern slope of the mountains in the north Indochina-southwest China region, and two secondary maxima, $N_{F}$, in the Tonkin Bay-northern South China Sea and the BOB-Myanmar regions. A population distribution of rainstorm genesis over these regions is digitized in the first column of Table 3. A majority of the East Asian rainstorm genesis occurs east of $100^{\circ} \mathrm{E}$, but about $1 / 6$ over the BOBMyanmar region. The dynamic impact of this land-sea differential radiative heating on rainstorm genesis will be addressed in later sections.

\section{b. Vertical locations of genesis and the spatial relationship between genesis and fronts}

The geneses of all identified rainstorms, whether over land or sea, only occur in the midtroposphere (700$500 \mathrm{hPa}$ ) (Fig. 6d). As with the genesis of the African easterly wave (e.g., Burpee 1972), there should be some special dynamic constraints of the monsoon circulation in Southeast/East Asia on the occurrence of rainstorm genesis. Shown in Figs. 5a and 5b, a typical rainstorm 
genesis (30 May 2008) in northern Vietnam is located farther west than the East Asian front. Is this null relationship between the genesis and the front true for all rainstorm geneses? To clarify this concern, distances between genesis locations over land (red dots) and sea (blue dots) in Figs. 6a and 6b, respectively, and tails of these possibly related fronts appearing on the JMA surface charts are shown in the scatter diagrams of Fig. 6f. Some identified rainstorms during certain stages of their life cycle may merge with fronts at downstream locations (solid/open triangles in Fig. 6e) east of their genesis locations. The relationships between rainstorm genesis from three regions and fronts, and the merger of these rainstorms and fronts are summarized in Table 3 in terms of the rainstorm population from these regions.

\section{Favorable environment for rainstorm genesis}

We focus on rainstorm genesis occurrence over two maximum regions (Fig. 6a): the northern Vietnamsouthwestern China region and the northern South China Sea. From a climatological perspective, we first examine the large-scale environment favorable for the occurrence of rainstorms in these two regions. The Charney-Stern instability criterion is then tested to determine whether the large-scale circulation over these two regions satisfy this criterion. Finally, the synoptic condition for a typical case of rainstorm genesis in each preferred region will be depicted and inferences of the rainstorm genesis mechanism substantiated with the diagnostic analysis of the vorticity and heat budgets.

\section{a. Large-scale monsoon circulation during the mei-yu season}

After monsoon onset in Southeast/East Asia, the monsoon circulation during the active phase (16 May15 June) (Fig. 7) is characterized by the following features. The upper-level strong westerlies migrate across the Tibetan Plateau and the tropical easterly jet develops around the southern rim of the Tibetan high. In contrast, juxtaposed with the western North Pacific subtropical high, the Asian continental thermal low embedded by the Tibetan Plateau is surrounded by the monsoon westerlies in South Asia and the monsoon southwesterlies in Southeast/East Asia.

The latitude-height cross section of the zonal wind component at $112.5^{\circ} \mathrm{E}$ (Fig. 7) shows the lowertropospheric monsoon westerlies across the northern part of Southeast Asia merge with the midtropospheric westerlies of the short-wave trough along the eastern seaboard of East Asia. As depicted by the 700- and 500-hPa streamline charts in Fig. 7, a confluence forms by these westerly flows over East Asia. To $700 \mathrm{hPa}$, this confluence forms a positive shear flow, extending from southwest China to the Yangtze River valley. The dynamic implication of this shear flow may be illustrated by the local Hadley circulation [depicted by $\left(v_{D},-\omega\right)$ ] at two longitudes $\left(104^{\circ}\right.$ and $\left.114^{\circ} \mathrm{E}\right)$ (Fig. 8), where $v_{D}$ is the meridional divergent wind. These are the locations where the maximum population of the rainstorm genesis (Fig. 6c) occurs in the middle troposphere. The upward branches of these local Hadley circulations are centered slightly north of $20^{\circ} \mathrm{N}$ across the shear line. The coincidence between the upward branch of the local Hadley circulation and the midtropospheric positive shear line provide a favorable environment for the genesis of rainstorms. This environmental condition may be coherently intensified by 1) the occurrence of cold surgelike northwesterlies east of the Tibetan Plateau in tandem with the eastward propagation of a midlatitude short-wave trough across the eastern seaboard of East Asia and 2) the monsoon southwesterlies across Indochina. The confluence of these two intensified flows in the midtroposphere is a favorable environment for rainstorm genesis and will be illustrated by case analyses in the next section.

\section{b. Dynamic instability}

As demonstrated by Burpee (1972), the genesis of African easterly waves occurring along the southern side of the African easterly jet is attributed to Charney-Stern instability (Charney and Stern 1962): a reversal of the meridional gradient $q_{y}$ of Ertel potential vorticity $q$ occurs along the south flank of a baroclinic jet and $q_{y}<0$ underneath this jet coupled with a maximum $\theta_{y}$ (meridional gradient of potential temperature $\theta$ ) near the surface (Thorncroft and Hoskins 1994).

It was previously illustrated that the western end of the positive monsoon shear flow (between northern Vietnam and eastern China) provides an environment convectively and dynamically favorable for rainstorm genesis. From a dynamic perspective, it is important to understand why the possible mechanism of rainstorm genesis exists in the midtroposphere. Suggested by the contrast between locations of rainstorm genesis (Figs. 6a,c) and the environmental circulation (Fig. 7) where the rainstorm geneses are embedded, the CharneyStern instability is a likely mechanism. The maximum rainstorm genesis occurrence (Fig. 6b) appears at two longitudes: $104^{\circ} \mathrm{E}$ over the northern Vietnam-southeastern China region and $114^{\circ} \mathrm{E}$ over the northern part of the South China Sea. The latitude-height cross sections of $\left(q_{y}, u\right)$ are shown in Figs. 8a and 8c. South of the maximum westerlies a sign change of $q_{y}$ occurs in the midtroposphere. Potential temperatures near the ground at both longitudes (Figs. 8b,d) exhibit a positive meridional 


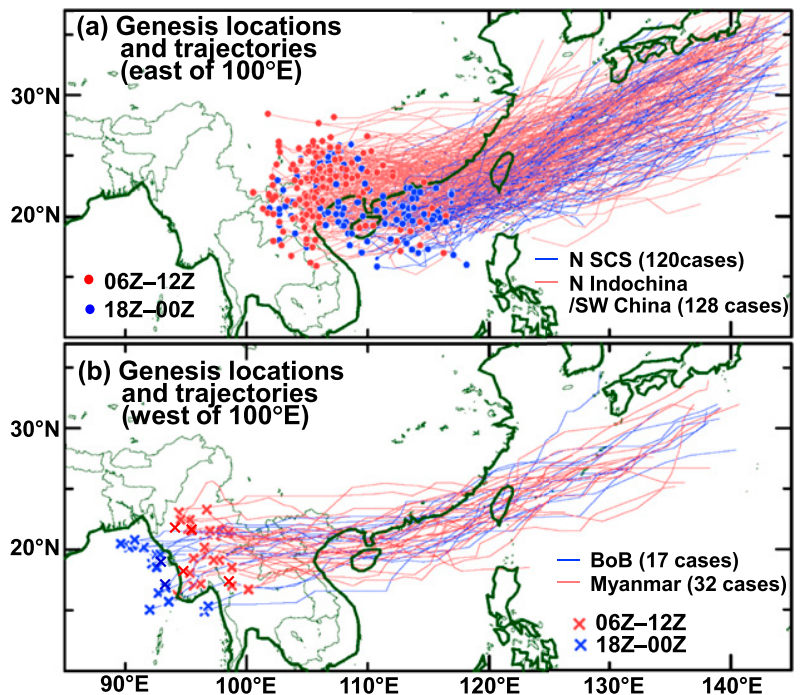

(c) Genesis population $\left(\mathrm{N}_{\mathrm{F}}\right)$ of rainstorm
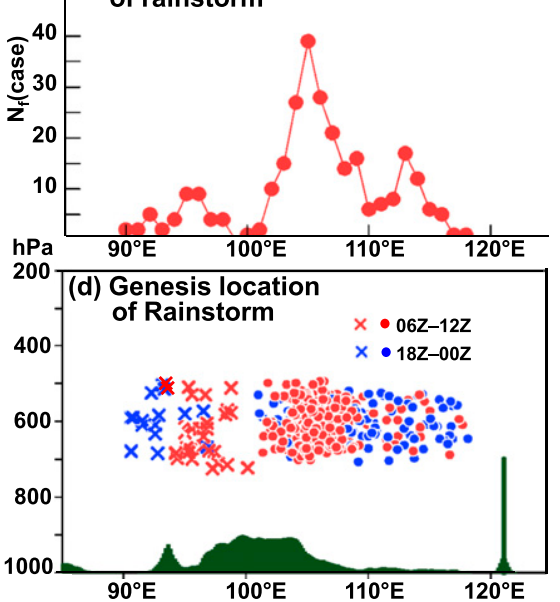

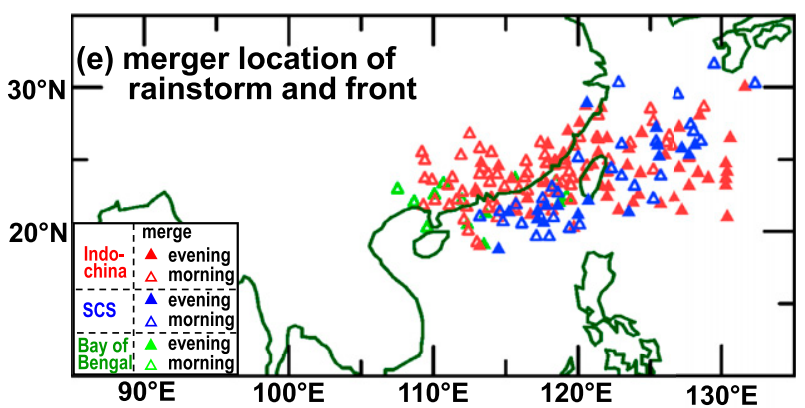

(f) Distance between RS and front tail
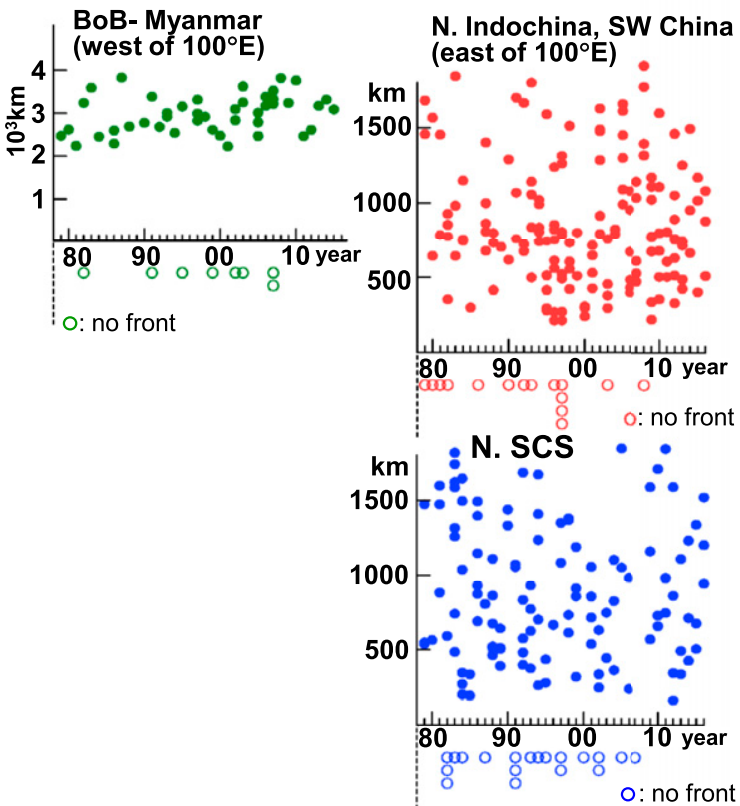

FIG. 6. Genesis statistics for rainstorms: (a) locations of genesis and trajectories for all identified rainstorms east of $100^{\circ} \mathrm{E}$. (b) As in (a), but for all identified rainstorms west of $100^{\circ}$ E. (c) Accumulated genesis occurrence for each degree of longitude. (d) Vertical locations of all identified geneses. (e) Merging locations (triangles) of rainstorms and East Asian fronts. (f) Distance between the genesis location and the tail of East Asian fronts for all identified rainstorms over the southwest China-northern Indochina region (0600-1200 UTC; red dots; land) and over the northern South China Sea (1800-0000 UTC; blue dots; sea). Red and blue dots in (a) represent genesis locations that occurred during late evening and early morning, respectively. The trajectories of rainstorms are indicated by color lines corresponding to their genesis in (a) and (b). The merging location of rainstorms and East Asian front in (e) is indicated by solid and open triangle in red (southwest China-northern Indochina rainstorm) and blue (northern SCS rainstorm).

gradient, $\theta_{y}>0$, slightly north of the latitude where $q_{y}$ changes its sign. These two conditions satisfy the CharneyStern instability criterion. The upward branches of the local Hadley circulations at the two longitudes (Figs. 8b,d) indicate the uplift of the air mass is coincident with the dynamically unstable zone, which facilitates rainstorm genesis.

\section{c. Case analysis}

Inferred from Figs. 4 and 5, the rainstorm genesis may occur when the northwesterly flow east of the Tibetan
Plateau is intensified by an eastward-propagating midlatitude synoptic short-wave trough located along the eastern seaboard of East Asia and the monsoon southwesterly flow is strengthened by the intensification of the monsoon circulation. To support this argument, the longitude-time diagrams of $700-\mathrm{hPa}$ wind vectors at $40^{\circ}$ and $25^{\circ} \mathrm{N}$ superimposed with rainstorm tracks (darkblue dots) are shown in Figs. 9a and 9b, respectively. These figures show rainstorm genesis occurs when pulses of northwesterly wind (blue arrow) and southwesterly flows (red arrow) begin to intensify. Thus, a positive 


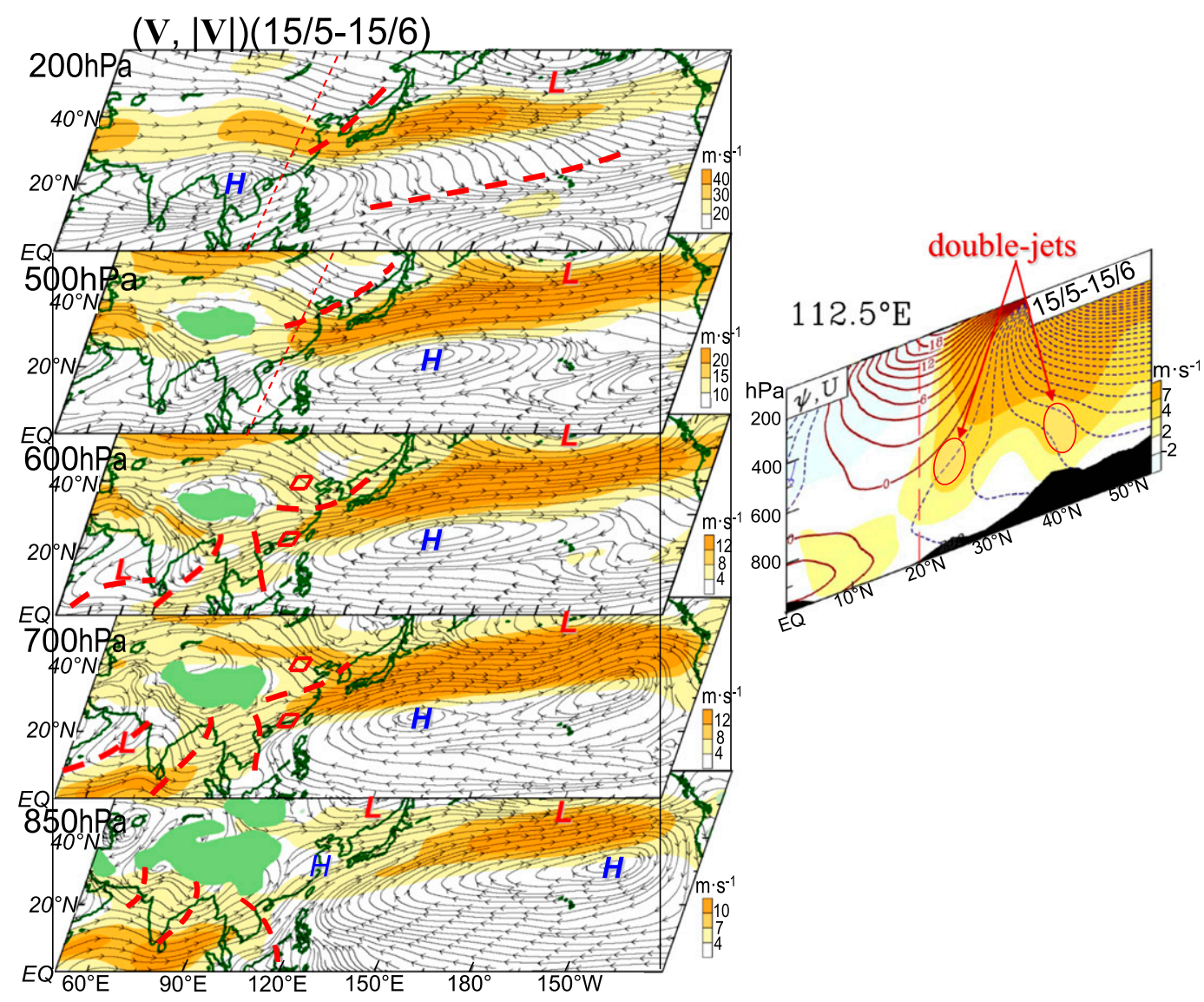

FIG. 7. Monthly (16 May-15 Jun) averaged large-scale circulation depicted by streamlines at different levels for the period 1979-2016. Isotachs are colored yellow and orange with the scale shown in the lower-right-hand corner of each panel. The trough line is marked by thick red-dashed line. Blue H and red L denote anticyclone and trough/cyclone, respectively. The latitude-height cross section of westerlies (golden color) and streamfunction (contours) at $112.5^{\circ} \mathrm{E}$ are shown in the right-hand panel. The scale for $u\left(112.5^{\circ} \mathrm{E}\right)$ is shown in the lower-right-hand corner of this panel. Departures of northwesterlies $\Delta \mathbf{V}_{\mathrm{NW}}$ in the northern red square and southeasterlies $\Delta \mathbf{V}_{\mathrm{SE}}$ in the southern red square from their corresponding climatological mean values are used to prepare Fig. 10.

shear flow is developed by the confluence of these two flows to establish an environment favorable for rainstorm genesis. Can the argument presented here be universally applicable to all 248 identified rainstorm geneses. Let $\Delta \mathbf{V}_{\mathrm{NW}}$ and $\Delta \mathbf{V}_{\mathrm{SW}}$ be departures from the mean Tibetan northwesterlies and the mean monsoon southwesterly flow at $700 \mathrm{hPa}$, respectively. All scatters (dots) showing wind departures are located over this region, where $\Delta \mathbf{V}_{\mathrm{NW}}>0$ and $\Delta \mathbf{V}_{\mathrm{SW}}>0$ in Fig. 10, imply the confluence of the intensified Tibetan northwesterly and monsoon southwesterlies in the midtroposphere is universally applicable for the identified rainstorm genesis. Thus, positive shear flow developed by the confluence of these two flows establishes an environment favorable for rainstorm genesis. Synoptic conditions for a typical rainstorm genesis in northern
Vietnam and another in the northern South China Sea are analyzed in detail below to illustrate the dynamic/ thermodynamic processes involved.

\section{1) CASE 1: RAINSTORM GENESIS IN NORTHERN VIETNAM ON 30 MAY 2008}

\section{(i) Synoptics}

On 30 May 2008, a well-developed deep 200-hPa short-wave trough over the eastern seaboard of northeast Asia was separated from the Southeast Asian anticyclone by the east Asian jet (Fig. 11a). Trailed by a ridge, this trough couples with a low-level, high-low couplet (Figs. 11b,c). A cold surgelike northeasterly flow, coupled with the upper-level northwesterlies ahead of the ridge, straddles the high and low centers of 
(a) $[\partial q / \partial y, \mathrm{u}]\left(104^{\circ} \mathrm{E}\right) \mathrm{Cl}: 2 \times 10^{-10} \mathrm{~K}^{\circ} \mathrm{cb}^{-1} \mathrm{~s}^{-1}$

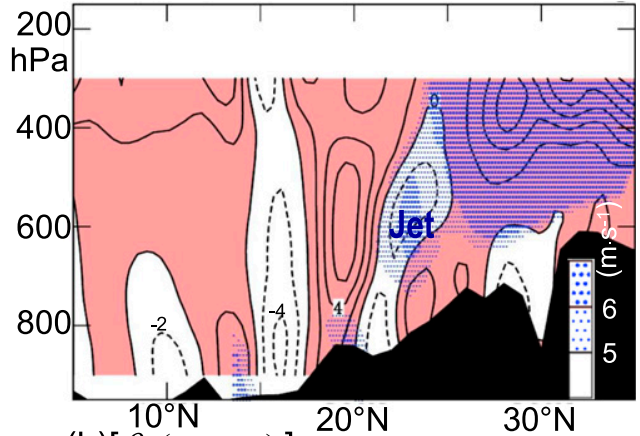

$(\mathrm{c})[\partial q / \partial y, \mathrm{u}]\left(114^{\circ} \mathrm{E}\right) \mathrm{Cl}: 2 \times 10^{-10} \mathrm{~K}^{\circ} \mathrm{Cb}^{-1} \mathrm{~s}^{-1}$

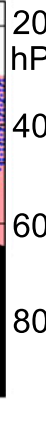

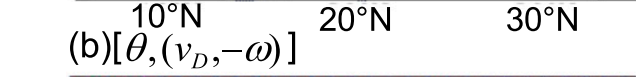
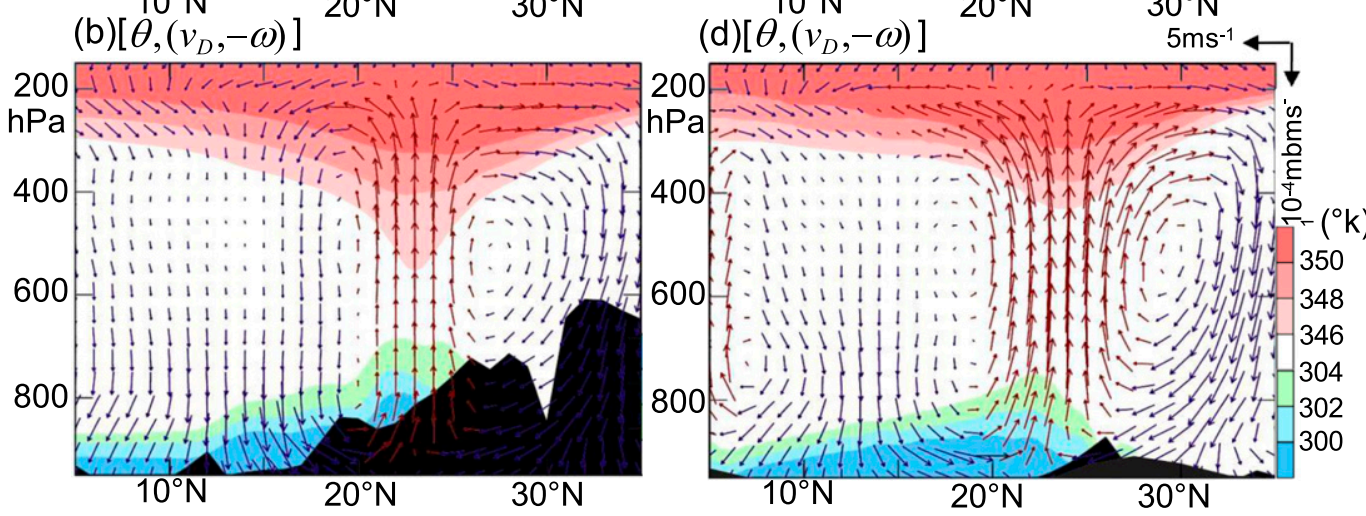

FIG. 8. Latitude-height cross sections of (a) $(\partial q / \partial y, u)\left(104^{\circ} \mathrm{E}\right)$, where $q$ is potential vorticity; (b) $\left[\theta,\left(v_{D},-\omega\right)\right]$ $\left(104^{\circ} \mathrm{E}\right)$; and (c),(d) the corresponding cross sections at $114^{\circ} \mathrm{E}$. The contour interval of $\partial q / \partial y$ and the scale of $u$ are shown at the top right of (a) and (c) and at the bottom right of (c), respectively. Note, $q$ is potential vorticity. Positive values of $\partial q / \partial y$ are shaded red. The color scale for $\theta$ is located at the bottom right of (d). All variables shown in this figure are averaged over the May-June period of 1979-2016.

this couplet. The confluence of the midtropospheric northwesterlies and the monsoon southwesterlies develop a cyclonic shear flow. The western end of this shear flow becomes a breeding ground for rainstorm genesis. This argument is confirmed by a low pressure region shown on the JMA surface chart marked by a red "L" (Fig. 11d), and by the IR image of MTSAT with an R2 symbol (Fig. 11e) in northern Vietnam. Additionally, the confluence depicted by the GFS analyses is validated by the wind vectors measured using radiosondes (Fig. 11f)—northwesterlies above $700 \mathrm{hPa}$ at Hohhot, China ( $\mathrm{ZBHH})$, and westerlies in the lower troposphere at King Park (45004). Significant dewpoint depression exists at Hohhot over the entire troposphere within the cold surgelike flow, but almost disappears in the midtroposphere at King Park. This thermal contrast indicates the midtroposphere within the cyclonic shear flow is convectively unstable. Another synoptic feature is that the rainstorm genesis and the JMA-analyzed front are separated by a distance of $1000 \mathrm{~km}$ (Fig. 11d). The rainstorm genesis occurs west and independent of the front. Apparently, this rainstorm is not evolved from the MCS associated with the East Asian front. The synoptic conditions for this Vietnam rainstorm genesis are typical.

\section{(ii) Heat and vorticity analyses}

The thermodynamic equation (e.g., Holton and Hakim 2013) is

$$
\frac{\partial T}{\partial t}+\mathbf{V} \cdot \nabla T-s_{p} \omega=\frac{1}{c_{p}} \dot{Q}, \quad \text { where } \quad s_{p}=-\frac{T}{\theta} \frac{\partial \theta}{\partial p} .
$$

If $\partial T / \partial t$ (local rate of change of temperature) is smaller than other thermodynamic processes in Eq. (1), this equation may be approximated by

$$
-s_{p} \omega \simeq \frac{1}{c_{p}} \dot{Q}-\mathbf{V} \cdot \nabla T
$$

As revealed from Eq. (2), vertical motion $(-\omega)$ can be initiated/maintained by diabatic heating $\left[\left(1 / c_{p}\right) \dot{Q}\right]$ and thermal advection $(-\mathbf{V} \cdot \nabla T)$. Note, diabatic heating $\left[\left(1 / c_{p}\right) \dot{Q}\right]$ may be evaluated in terms of the residual term in Eq. (1) (e.g., Chen and Baker 1986). All thermodynamic processes involved with the $\mathrm{R} 2$ rainstorm genesis (Fig. 12) are highlighted by the following features:

- The diabatic heating $\left(1 / c_{p}\right) \dot{Q}>0$ (Fig. 12b) at the genesis location of rainstorm $\mathrm{R} 2$, and over its vicinity, 


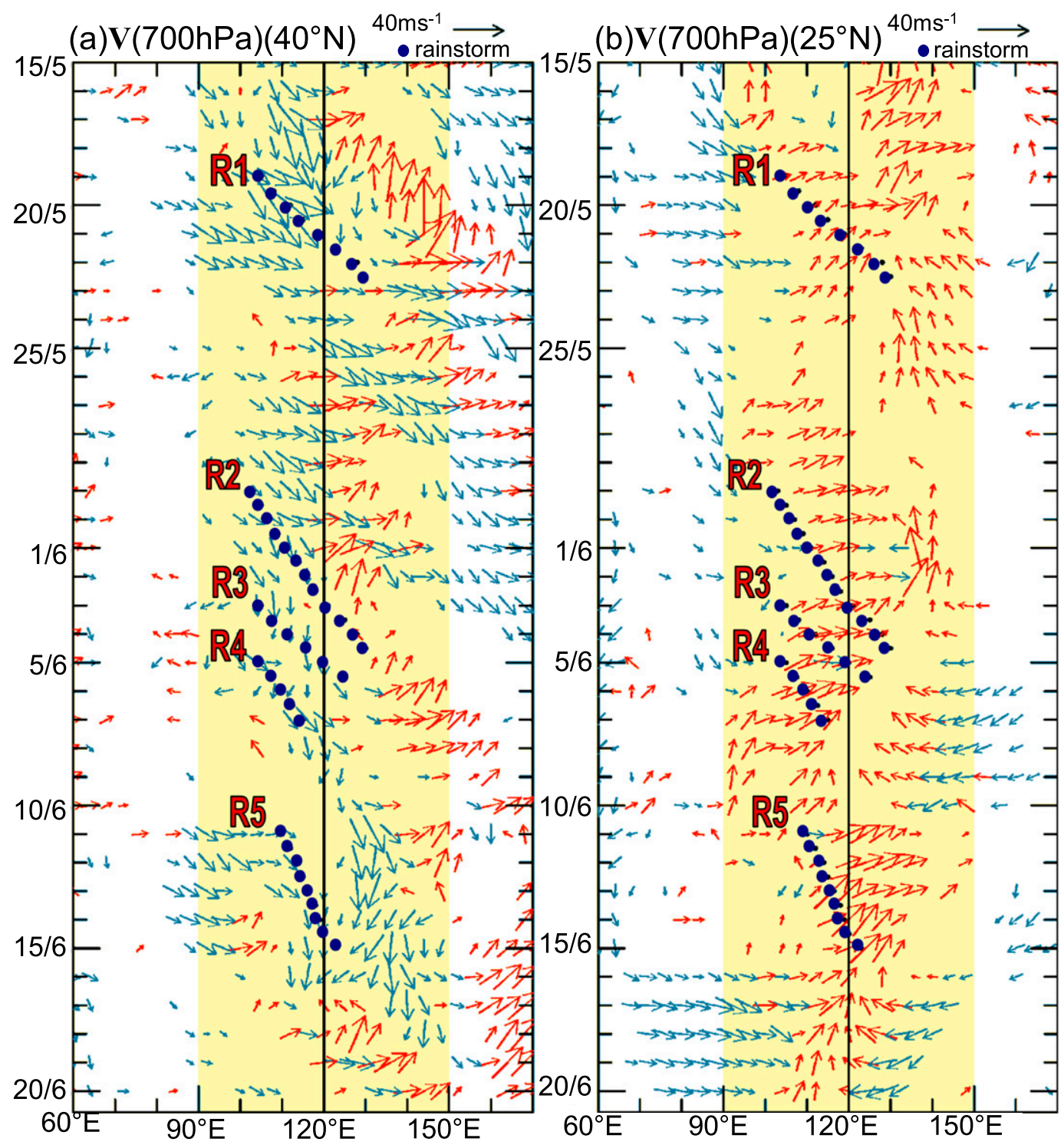

FIG. 9. The longitude-time diagram of $700-\mathrm{hPa}$ wind vectors $\mathbf{V}\left(700 \mathrm{hPa}\right.$ ) at (a) $40^{\circ}$ and (b) $25^{\circ} \mathrm{N}$. The longitudinal locations of all identified rainstorms (indicated by R1-R5) during the period midMay-June 2008 are marked by dark-blue dots. Northwesterly or northerly (southwesterly or southerly) wind vectors are represented by blue (red) vectors. The scale of wind vectors is shown on the top right of each panel.

while $\partial T / \partial t$ (Fig. 12e) is small/negative. The magnitude ratio $|\partial T / \partial t| /\left|\dot{Q} / c_{p}\right|$ around the $\mathrm{R} 2$ genesis location (Fig. 12d) is $<10 \%$.

- Shown in Fig. 11c, rainfall occurs along the front, but becomes insignificant over the narrow trough (accommodating the rainstorm embryo of $\mathrm{R} 2$ with the symbol L) west of the front.

- The close resemblance between the distributions of $\dot{Q} / c_{p}$ and $-s_{p} \omega$ (Fig. 12f) strongly indicates that the vertical motion along the narrow trough and the front are a response of the air mass to the diabatic heating generated from the heat of condensation from water vapor, supplemented by the sensible heat advection, $-\mathbf{V} \cdot \nabla T$ (Fig. 12a).

It is inferred from the approximated heat budget [Eq. (2)] that the spinup process for the $\mathrm{R} 2$ genesis is driven through the diabatic cooling $\left(-S_{p} \omega\right)$ by the collaborative effect of $\left(1 / c_{p}\right) \dot{Q}$ and $-\mathbf{V} \cdot \nabla T$ (Fig. 12c) along the positive shear zone and the front through the vortex stretching illustrated in terms of the vorticity equation. 


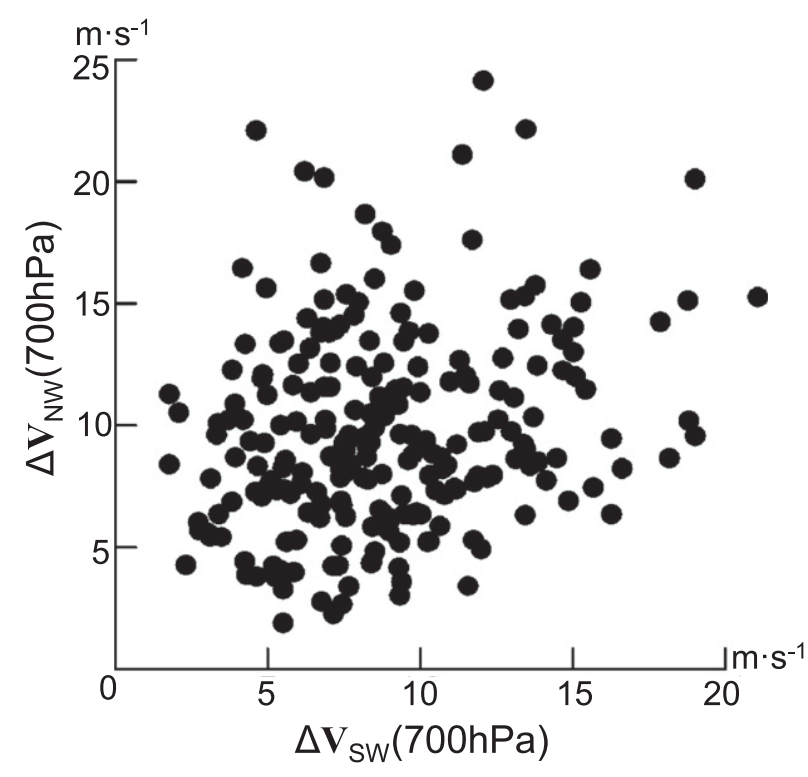

FIG. 10. A scatter (black dot) diagram formed using northwesterly departures from the climatological values $\Delta \mathbf{V}_{\mathrm{NW}}(700 \mathrm{hPa})$ averaged over the red square (centered at $40^{\circ} \mathrm{N}, 112.5^{\circ} \mathrm{E}$ ) in Fig. 7 , northeast of the Tibetan Plateau and monsoon southwesterly departures from the climatological value $\Delta \mathbf{V}_{\mathrm{Sw}}(700 \mathrm{hPa})$ averaged over the red square (centered at $20^{\circ} \mathrm{N}, 112.5^{\circ} \mathrm{E}$ ) in Fig. 7 in southern China.

The complete vorticity equation (e.g., Holton and Hakim 2013) can be written as follows

$$
\begin{aligned}
\frac{\partial \zeta}{\partial t}= & \underbrace{-\mathbf{V} \cdot \nabla(\zeta+f)}_{\text {VAD }} \underbrace{-(\zeta+f) \nabla \cdot \mathbf{V}}_{\text {VST }} \\
& \underbrace{-\omega \frac{\partial \zeta}{\partial p}-\frac{\partial \omega}{\partial x} \frac{\partial v}{\partial p}+\frac{\partial \omega}{\partial y} \frac{\partial u}{\partial p}+\frac{\partial F_{y}}{\partial x}-\frac{\partial F_{x}}{\partial y}}_{\text {VRE }},
\end{aligned}
$$

where $\zeta_{t}$ (tendency of relative vorticity), VAD (advection of absolute vorticity), VST (stretching of absolute vorticity), and VRE (residual term of the vorticity equation) for the genesis of a $\mathrm{R} 2$ rainstorm are shown in Fig. 13. VRE (Fig. 13d) is smaller in magnitude than individually with the other two dynamic processes: VAD and VST. The magnitude of $\zeta_{t}$ (Fig. 13a) is close to $-(\mathrm{VAD}+\mathrm{VST})$ (Fig. 13d), because VRE/ $\zeta_{t}$ around the R2 genesis location is smaller than $10 \%$. Thus, Eq. (3) can be simplified into Haynes and McIntyre's (1987) flux form for the vorticity equation:

$$
\zeta_{t} \simeq-\nabla \cdot[\mathbf{V}(\zeta+f)]
$$

Although the rainstorm propagation mechanism through VAD is not explicitly provided, $\zeta_{t}$ is simply a response of the local vorticity tendency to the convergence of total vorticity flux. Another insight for the spinup of the R2 genesis can be revealed from the comparison between dynamic processes of VAD (Fig. 13b) and VST (Fig. 13c) over the area around the genesis location (indicated by the intersection of two thin red lines). Clearly, $\zeta_{t}$ is primarily contributed by VST through the vertical motion driven by diabatic heating illustrated by the heat budget of the R2 genesis shown in Fig. 12.

\section{2) CASE 2: RAINSTORM GENESIS IN THE NORTHERN SOUTH CHINA SEA ON 4 MAY 1994}

\section{(i) Synoptics}

A secondary maximum of rainstorm genesis occurrence frequency appears in the northern South China Sea (Fig. 6b). The synoptic condition of a typical rainstorm genesis for this region at 0600 UTC 4 May 1994 is shown in Fig. 14. An upper-level short-wave trough (thick red line) located along the eastern seaboard of northeast Asia was separated from the east-westoriented South-Southeast Asian anticyclone by the subtropical westerly jet (Fig. 14a). The upper-level ridge-trough synoptic system was coupled with a lowlevel high-low couplet straddled by a cold surgelike northwesterly flow (Figs. 14b,c). Along the southeastern China coast in the low troposphere, the confluence of the midtropospheric northwesterly flow and the monsoon southwesterly flow form a narrow trough slightly west of Hainan Island (Figs. 14b,c). This narrow trough is verified with the radiosonde observations of wind vectors at Taiyuan, China (ZBYN), and King Park below $500 \mathrm{hPa}$ (Fig. 14f). The northwesterly flow is dry (significant dewpoint depression), while the monsoon southwesterly flow is moist (dewpoint depression $\sim 0^{\circ} \mathrm{C}$ ). A merger of these two air masses along this narrow trough made the flow convectively and dynamically unstable. Identified by PERSIANN precipitation ( $\mathrm{R}$ in Fig. 14c) and the TBB image of GMS observations ( $R$ in Fig. 14e), a rainstorm genesis was located at approximately $400 \mathrm{~km}$ west of the tail of the East Asian front (shown on the JMA surface chart in Fig. 14d).

\section{(ii) Heat and vorticity budget analysis}

As the R2 rainstorm genesis in northern Vietnam, $(\partial T / \partial t) /\left(1 / c_{p}\right) \dot{Q}$ (Fig. $15 \mathrm{~d}$ ) is $<<1$ at the genesis location of the SCS R rainstorm and over its vicinity. The close resemblance in the spatial distribution of $\left[\left(1 / c_{p}\right) \dot{Q}(-\mathbf{V} \cdot \nabla T)\right]$ (Fig. 15c), and $-s_{p} \omega$ (Fig. 15f) reveals the vertical motion along the narrow trough and the front is a response of the lower-tropospheric flow to the diabatic heating generated by the heat condensation of the cloud droplet (Fig. 15b), supplemented by the weak sensible heat advection $-\mathbf{V} \cdot \nabla T$ (Fig. 15a). To satisfy mass conservation, the low-level convergence and 

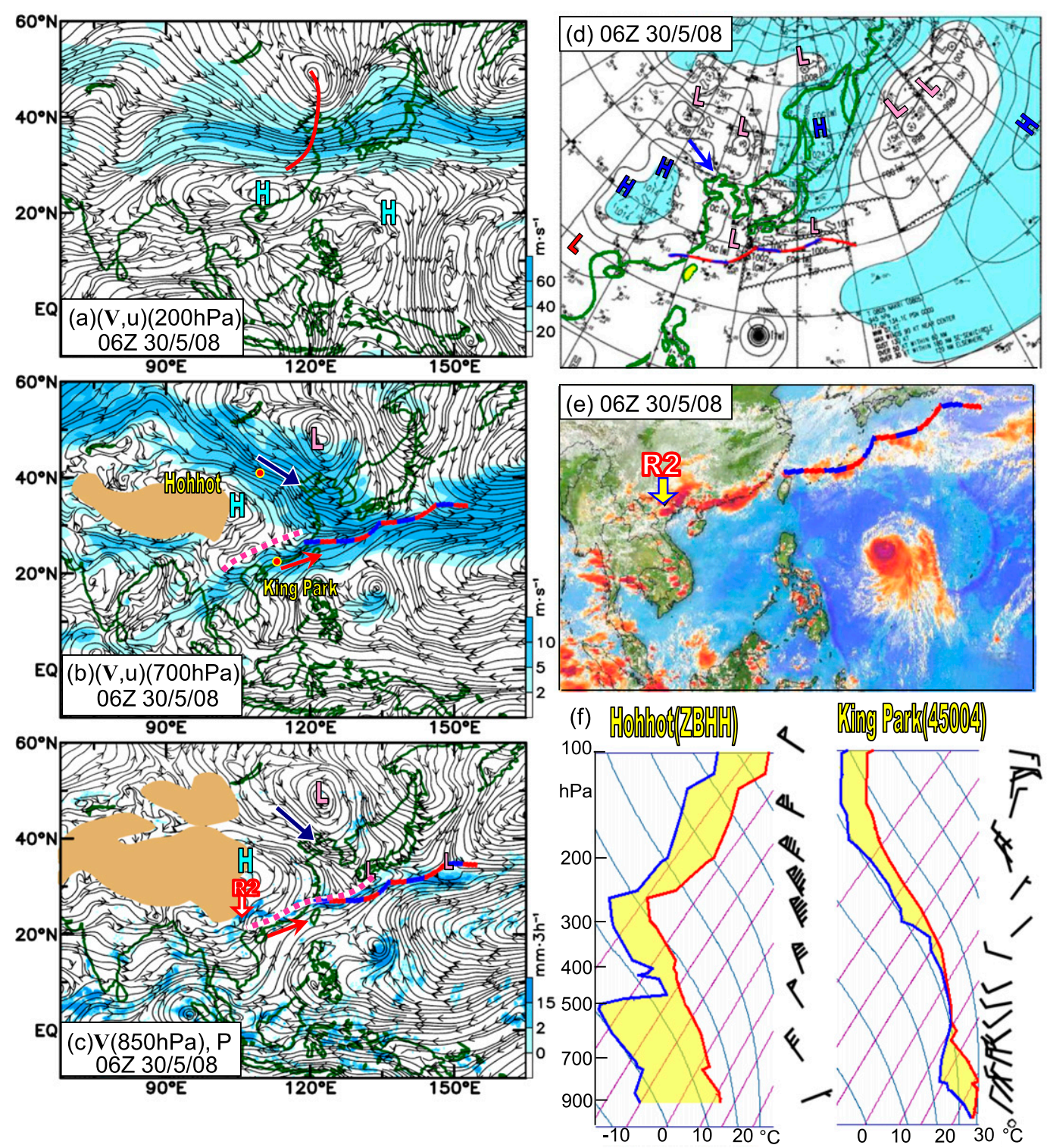

FIG. 11. Synoptic conditions of the rainstorm genesis that occurred at 0600 UTC 30 May 2008: (a) 200-hPa streamline chart superimposed with isotachs (blue) and a trough line (thick red line). (b) As in (a), but at $700 \mathrm{hPa}$ and superimposed with a front, a shearline (red-dashed line), a dark-blue arrow (northwesterly flow) with a blue $\mathrm{H}$ and red L to the arrow's SW and NE side, respectively, and a red arrow (southwesterly flow). (c) As in (b), but at $850 \mathrm{hPa}$ with TRMM precipitation (blue) and the genesis location of rainstorm 2 (R2). (d) JMA surface analysis chart. (e) IR image of MTSAT superimposed with front and the R2 genesis location. (f) Radiosonde observations at Hohhot (ZBHH) and King Park (45004). Vertical profiles in (f) are dewpoint (blue) and temperature (red).

upper-level divergence of the air mass should be developed by the upward motion generated by diabatic heating associated with the rainstorm genesis. The ratio, $\mathrm{VRE} / \zeta_{t}<<1$, is around the genesis location of the SCS rainstorm (Fig. 16f). Because the VAD process (Fig. 16b) is insignificant around this rainstorm genesis location, the major contribution to $\zeta_{t}$ comes from VST (Fig. 16c). Shown in Fig. 16d it matches well with $\zeta$, the spinup process for the SCS rainstorm genesis apparently is caused by the convergence of total vorticity flux driven by the vertical motion (Fig. 15f), as the thermodynamic and dynamic processes illustrated for the genesis of case 1 .

\section{d. Summary}

The confluence of the cold surgelike northwesterly flow east of the Tibetan Plateau and the monsoon southwesterly flow develops a narrow trough. The 

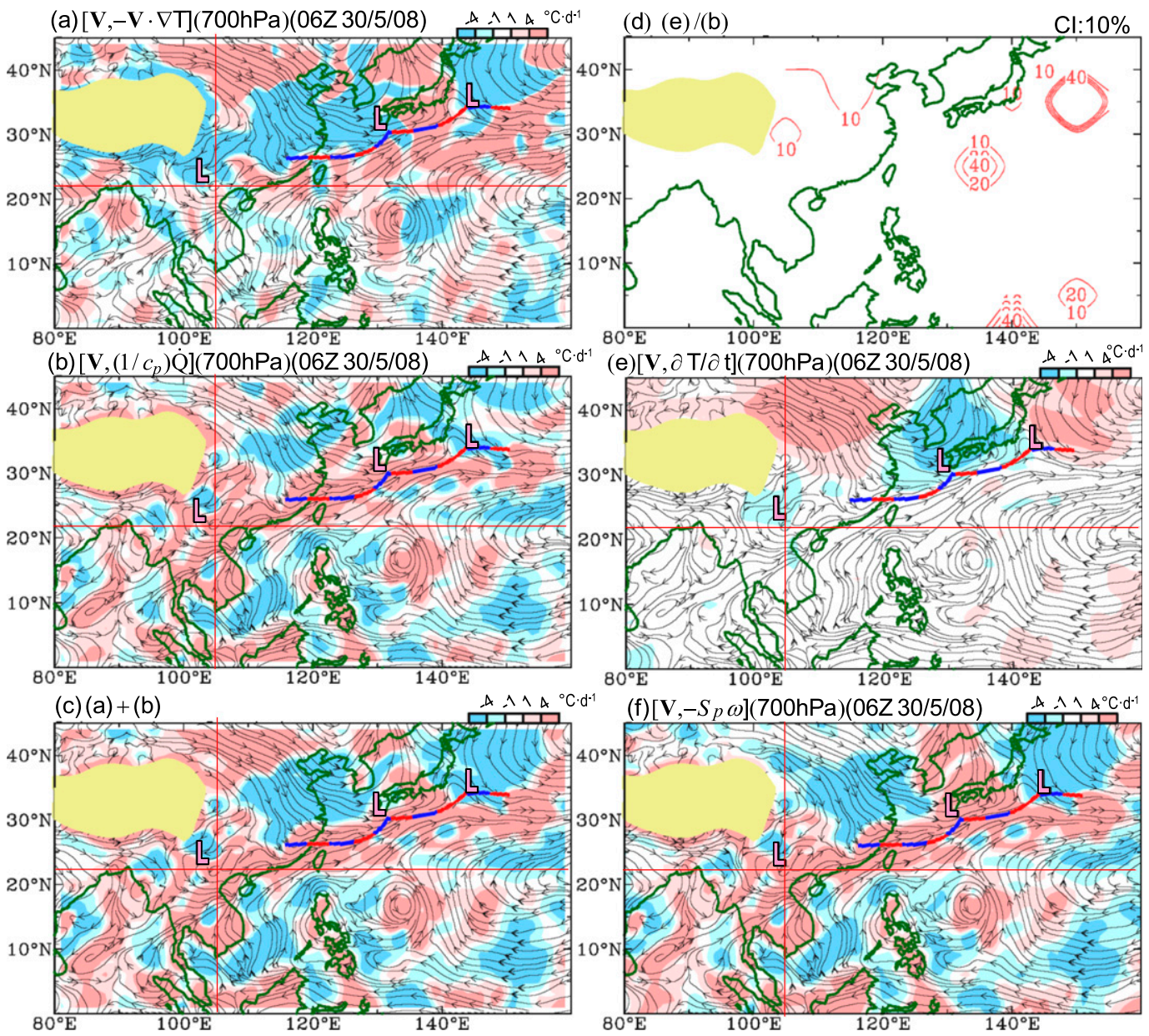

FIG. 12. The 700-hPa heat budget analysis for the rainstorm R2 genesis occurrence at 0600 UTC 30 May 2008: (a) the 700-hPa streamline charts superimposed with $-\mathbf{V} \cdot \nabla T(700 \mathrm{hPa}),(\mathrm{b})\left(1 / c_{p}\right) \dot{Q}$, (c) $[(\mathrm{a})+(\mathrm{b})]$, (d) $[(\mathrm{e}) /(\mathrm{b})]$, (e) $\partial T / \partial t(700 \mathrm{hPa})$, and (f) $-s_{p} \omega(700 \mathrm{hPa})$. The genesis location marked by red $\mathrm{L}$ and front are added at each panel, except for (d). Color scales for all variables are shown at the top right of each panel. Two thin red lines are added on each panel to indicate the identified location of rainstorm genesis.

western end of this narrow trough becomes a breeding ground for rainstorms during the active phase of the Southeast/East Asian summer monsoon. The spinup of air mass through vortex stretching/convergence of total vorticity flux at the western end of this trough makes rainstorm genesis possible by upward vertical motion. To ascertain these findings are representative for all identified rainstorm geneses, two three-dimensional scatter diagrams are shown in Fig. 17:

(i) The heat budget analysis (Fig. 17a): Upward vertical motion $(-\omega>0)$ in the vicinity of rainstorm genesis is primarily maintained by diabatic heating, $\dot{Q} / c_{p}$.

(ii) The vorticity budget analysis (Fig. 17b): In the vicinity of rainstorm genesis, the spinup of the rainstorm embryo $\left(\zeta_{t}>0\right)$ is induced/maintained by an upward vertical motion $(-\omega>0)$ through positive vortex stretching $[-(\zeta+f) \nabla \cdot \mathbf{V}>0]$ or convergence of total vorticity flux $-\nabla \cdot[(\zeta+f) \mathbf{V}]$.

\section{Diurnal variation of rainstorm genesis}

The mechanism of rainstorm genesis was illustrated from two perspectives: 1) large-scale environmental flow favorable for rainstorm genesis and 2) dynamic instability of the environmental flow. As shown in Fig. 6a, most rainstorm geneses on land occur in the late evening and at sea in the early morning. It was inferred from this diurnal variation in the timing preference of rainstorm genesis is regulated by the diurnal variation of the largescale monsoon circulation in response to the land-sea thermal contrast and the diurnal variation of dynamic instability of the environmental flow. 

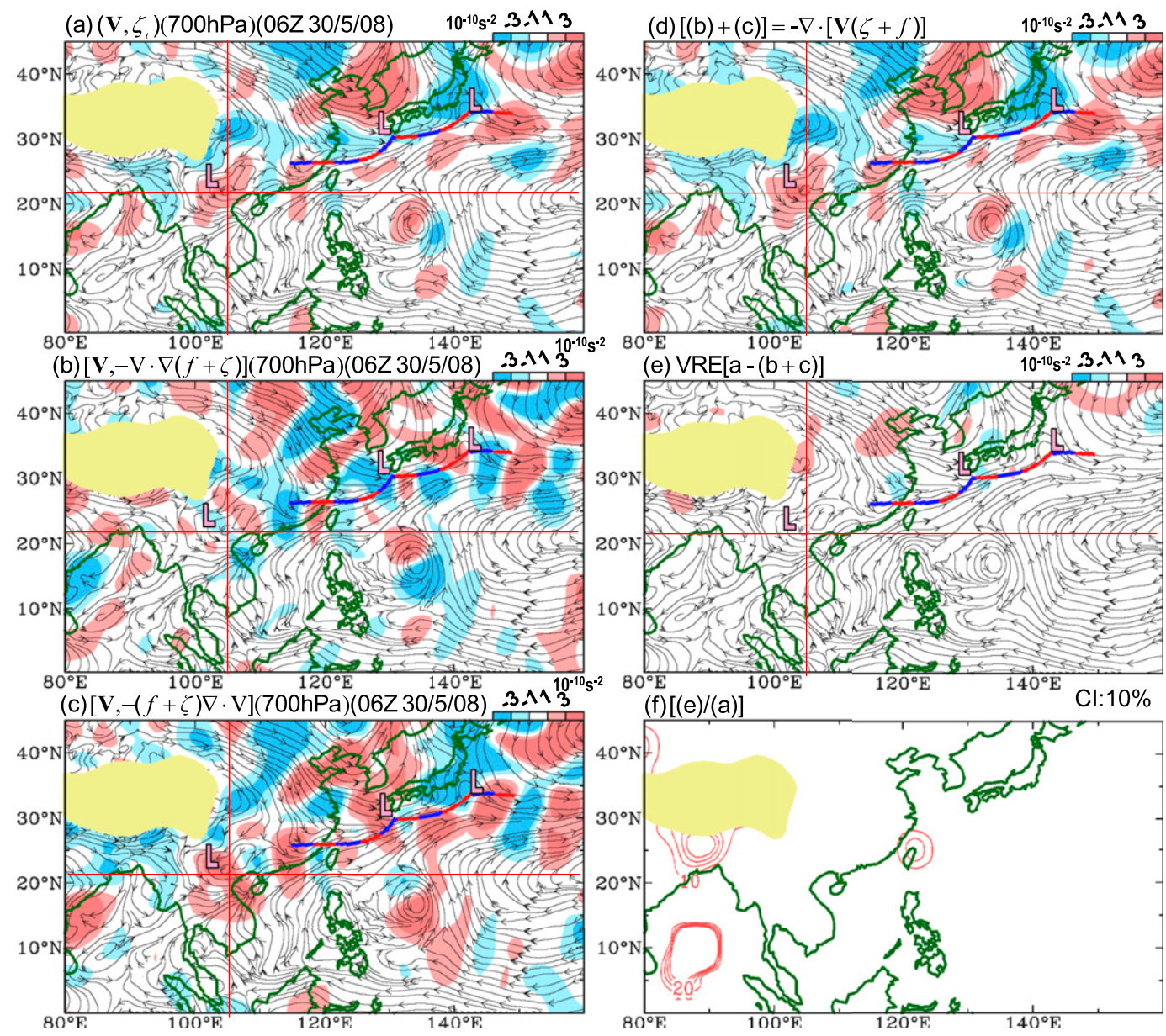

FIG. 13. The 700-hPa vorticity budget analysis at the geneses occurrence of rainstorm R2 (0600 UTC 30 May 2008): The 700-hPa streamline charts superimposed with (a) $\zeta_{t}(700 \mathrm{hPa}),(\mathrm{b})-\mathbf{V} \cdot \nabla(\zeta+f)(700 \mathrm{hPa}),(\mathrm{c})-(\zeta+f) \nabla \cdot \mathbf{V}(700 \mathrm{hPa})$, (d) $[(\mathrm{b})+(\mathrm{c})]$, (e) $\operatorname{VRE}\{(\mathrm{a})-[(\mathrm{b})+(\mathrm{c})]\}$, and (f) [(e)/(a)] without the 700-hPa streamline chart. As in Fig. 12, two thin red lines are added on each panel to indicate the identified location of rainstorm.

\section{a. Diurnal variation of the large-scale circulation in response to the land-sea thermal contrast}

The direct atmospheric response to diabatic heating is the development of divergent circulation. Because vertical motion is part of divergent circulation, modulation of the divergent circulation through the land-sea thermal contrast should be reflected by vertical motion. As shown in section 4 , rainstorm genesis is facilitated by vertical motion through vortex stretching/convergence of vorticity flux. Thus, the timing preference of the rainstorm genesis should be regulated by the diurnal variation of the landsea thermal contrast through the divergent circulation. This inference can be substantiated by diagnostic analyses, including maintenance of divergent circulation by diabatic heating (Chen and Yen 1991 a,b) and the streamfunction tendency by vortex stretching (Sanders 1984; Chen and Chen 1997).
Divergent circulation can be well portrayed with velocity potential $\chi$, while divergent winds can be measured with horizontal gradients of $\chi$ (i.e., $\mathbf{V}_{D}=\nabla \chi$ ). The maintenance of the divergent circulation by diabatic heating $\dot{Q}$ is illustrated by the $\chi$-maintenance equation, a combination of the continuity and the thermodynamic equations:

$$
\begin{aligned}
\chi= & \nabla^{-2}\left[\frac{\partial}{\partial p}\left(\frac{\partial T}{\partial t}+\mathbf{V} \cdot \nabla T\right)\right] \\
& +\nabla^{-2}\left\{\frac{\partial}{\partial p}\left[\frac{1}{\sigma c_{p}}\left(\frac{T}{\theta} \dot{Q}\right)\right]\right\}, \\
\chi_{\dot{Q}} &
\end{aligned}
$$

where $c_{p}, T, \theta, p$, and $\mathbf{V}$ are specific heat with constant pressure, temperature, potential temperature, pressure, and velocity vector, respectively. The diurnal component $(\frown$ ) of the divergent circulation is maintained by 

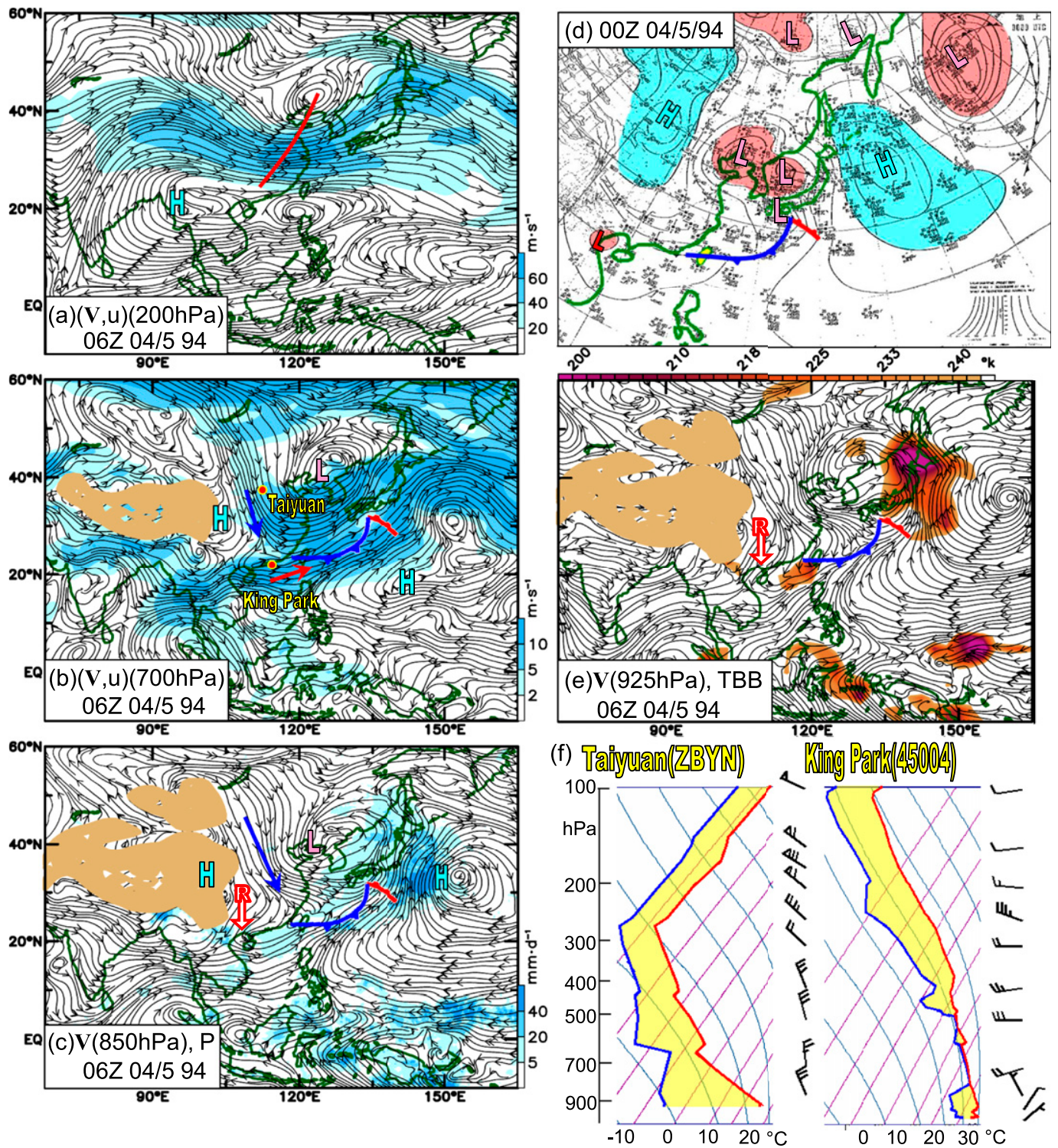

FIG. 14. (a)-(f) As in Fig. 11, but for 4 May 1994 with PERSIANN precipitation in (c), and TBB, instead of IR, is used to generate images of convective clouds in (e). The genesis location of this rainstorm is indicated with a redopen arrow marked by R. One sounding station was replaced by Taiyuan.

$$
\widehat{\chi}=\widehat{\chi}_{H}+\widehat{\chi}_{\dot{Q}} .
$$

The relationship between vorticity $\zeta$ and streamfunction $\psi$ is $\zeta=\nabla^{2} \psi$ or $\psi=\nabla^{-2} \zeta$. The streamfunction budget equation (i.e., an inverse Laplace transformation of the vorticity equation) may be written as

$$
\underset{\psi_{t}}{\frac{\partial \psi}{\partial t}}=\nabla_{\psi_{A}}^{-2}[-\mathbf{V} \cdot \nabla(\zeta+f)]+\nabla^{-2}\left[\underset{\psi_{\chi}}{\left.(\zeta+f) \frac{\partial \omega}{\partial p}\right]},\right.
$$

where $\partial \omega / \partial p=-\nabla \cdot \mathbf{V}$ (the continuity equation). According to the Helmholtz theorem, the horizontal atmospheric flow can be split into divergent and rotational flows, $\mathbf{V}=\mathbf{V}_{D}+\mathbf{V}_{R}$. Because $\left|\mathbf{V}_{D}\right| /\left|\mathbf{V}_{R}\right|<<1$, the horizontal atmospheric flow is primarily depicted by the rotational component. Maintenance of the diurnal component of the rotational flow may be expressed as

$$
\widehat{\psi}_{t}=\widehat{\psi}_{A}+\widehat{\psi}_{\chi} .
$$

Presumably, $\zeta / f$ ( $f=$ planetary vorticity $)<<1$ is true for the diurnal component of global circulation around $20^{\circ} \mathrm{N}$. For the global $\widehat{\psi}$ field, the streamfunction tendency caused by the vorticity advection $\widehat{\psi}_{A}$ may not be significant, and Eq. (8) may be approximated as 

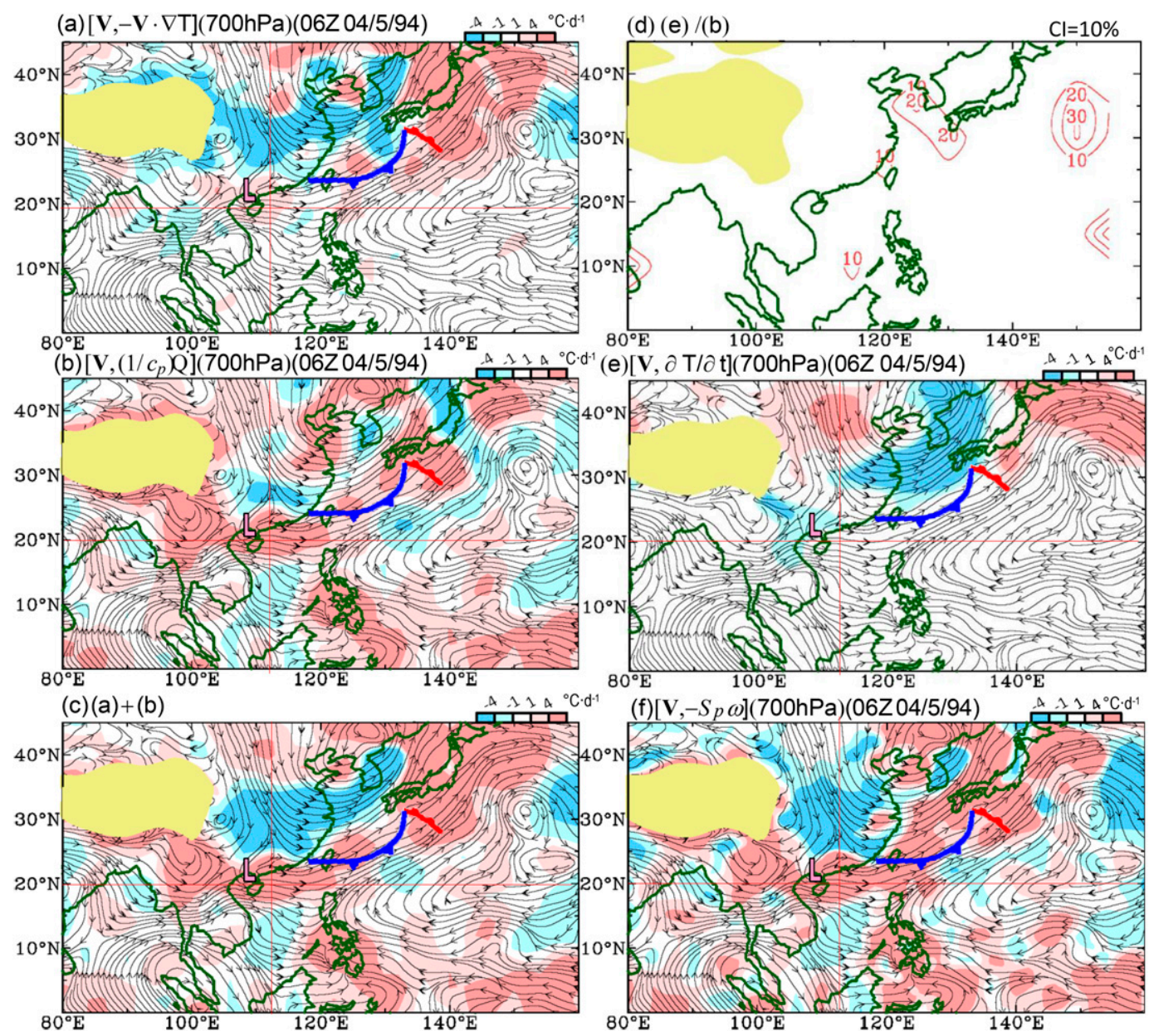

FIG. 15. As in Fig. 12, but the rainstorm genesis occurred at 0600 UTC 4 May 1994.

$$
\widehat{\psi}_{t} \simeq \nabla^{-2}(-f \nabla \cdot \widehat{\mathbf{V}}) .
$$

Assuming $f=f_{o}$ (constant), the diurnal mode of the streamfunction budget may be expressed as

$$
\widehat{\psi}_{t} \simeq-f_{o} \widehat{\chi} .
$$

As shown in Eqs. (9) and (10), the diurnal mode of the atmospheric rotational circulation is largely driven by its divergent circulation.

The timing preference of rainstorm genesis between land and sea (Fig. 6a) will be illustrated with Eqs. (6) and (8). Over the analysis domain, the variance of $\chi \chi \chi(850 \mathrm{hPa})$ contributed by $\hat{\chi}_{H}(850 \mathrm{hPa})$ and $\widehat{\chi}_{\dot{Q}}(850 \mathrm{hPa})$ are $8 \%$ and $92 \%$, respectively. An empirical orthogonal function (EOF) analysis was then performed with $\widehat{\chi}_{\dot{Q}}(850 \mathrm{hPa})$ to illustrate the maintenance of the divergent circulation by diabatic heating. The first two principal eigenmodes, including eigenvectors and time series of eigencoefficients, are shown in the left-hand column of Fig. 18. The land-sea thermal contrast is well portrayed by the first eigenvector $\mathrm{E} 1$ [ $\left.\widehat{\chi}_{\dot{Q}}(850 \mathrm{hPa})\right]$, which explains $63 \%$ of the variance of $\hat{\chi}_{\dot{Q}}(850 \mathrm{hPa})$. The time series of this eigenmode's eigencoefficient $\mathrm{C} 1\left[\widehat{\chi}_{\dot{Q}}(850 \mathrm{hPa})\right]$ reaches its maximum at 0300 UTC and minimum at 1500 UTC. Surprisingly, north-south differential heating also plays an important role, as depicted by the second eigenvector E2 $\left[\widehat{\chi}_{\dot{Q}}(850 \mathrm{hPa})\right]$, which explains $35.5 \%$ of the variance of $\hat{\chi}_{\dot{Q}}(850 \mathrm{hPa})$, with its maximum eigenvalue $\mathrm{C} 2$ $\left[\widehat{\chi}_{\dot{Q}}(850 \mathrm{hPa})\right]$ occurring at $0900 \mathrm{UTC}$ and its minimum eigenvalue occurring at 2100 UTC.

The EOF analysis of $\widehat{\chi}(850 \mathrm{hPa})$ is shown in the right-hand column of Fig. 18. Temporal variations of the eigencoefficient time series of these two $(850 \mathrm{hPa})$ eigenmodes (Fig. 18d) and the spatial structure for the two eigenvectors of $\widehat{\chi}(850 \mathrm{hPa})$ (Figs. 18e,f) resemble the corresponding $\widehat{\chi}_{\dot{Q}}$ eigenmodes (Figs. 18b,c). Apparently, the diurnal variation of the regional divergent circulation is a response of the Asian monsoon system to both the land-sea thermal contrast and the 

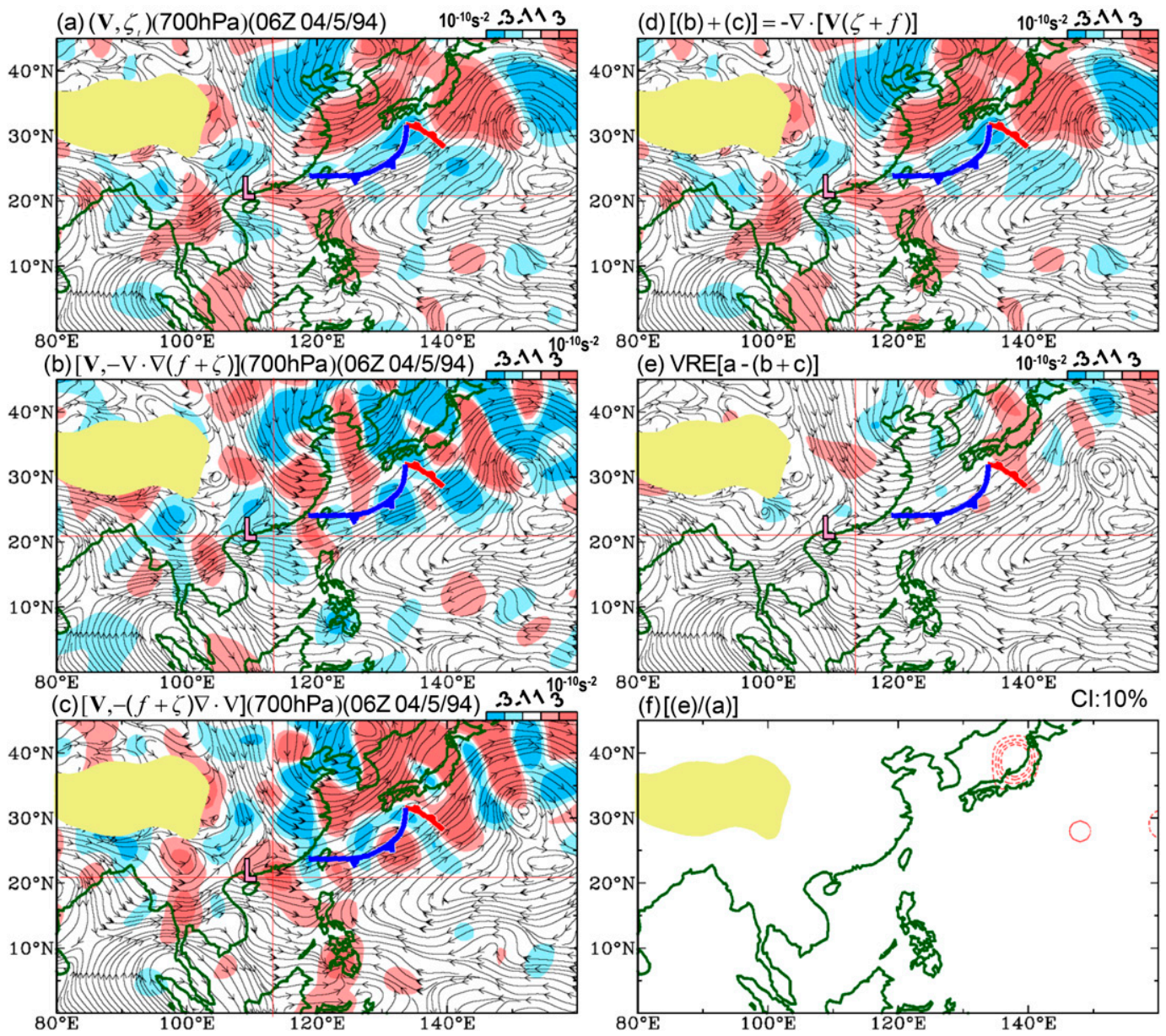

FIG. 16. As in Fig. 13, but the rainstorm genesis occurred at 0600 UTC 4 May 1994.

north-south differential heating. A daily clockwise rotation of $\widehat{\chi}(850 \mathrm{hPa})$ around the Asian continent and the western North Pacific is formed by a combination of its two eigenmodes (not shown).

The clockwise rotation of $\widehat{\chi}(850 \mathrm{hPa})$ over the Asian continent leads to the formation of the east-west circulation $\left(\widehat{u}_{D},-\widehat{\omega}\right)\left(20^{\circ} \mathrm{N}\right)$ between land and ocean shown in Fig. 19. At 1200 UTC (late evening, the left-hand column of Fig. 19), the upward branch of the east-west circulation over Indochina is coupled with low-level convergence and upper-level divergence, while the downward branch of the east-west circulation in the South China Sea is linked with low-level divergence and upper-level convergence. The structure of this east-west circulation facilitates the occurrence of rainstorm genesis over the northern Vietnam-southwestern China region and hinders it over the northern South China Sea. At 0000 UTC (early morning, the right-hand column of Fig. 19), the land-sea contrast of vertical motion, the associated divergent circulation, and the rainstorm genesis occurrence frequency are reversed.

Next, we shall explore dynamically how this diurnal variation in vertical motion modulates the rainstorm genesis activity between land and ocean. Using Eq. (9) or (10), we argued the diurnal variation of the regional streamfunction follows the diurnal variation of velocity potential across the Indochina-southwestern China region and the northern part of the South China Sea. The diurnal component of the streamfunction budget at 0900 UTC (1600 LST) and 2100 UTC (0400 LST) are shown in Fig. 20. The variances of $\widehat{\psi}_{t}(850 \mathrm{hPa})$ (computed by the central finite-difference approach) explained by $\psi_{A}(850 \mathrm{hPa})$ and $\widehat{\psi}_{\chi}(850 \mathrm{hPa})$ are $8 \%$ and $92 \%$, respectively. Thus, $\widehat{\psi}_{t}(850 \mathrm{hPa})$ is close to $\widehat{\psi}_{\chi}(850 \mathrm{hPa})$ in spatial structure and magnitude. The negative $\widehat{\psi}_{t}(850 \mathrm{hPa}) /$ $\widehat{\psi}_{\chi}(850 \mathrm{hPa})$ center over Indochina and southern China at 0900 UTC facilitates the evening rainstorm genesis. During this time, values for $\widehat{\psi}_{t}(850 \mathrm{hPa}) / \widehat{\psi}_{\chi}(850 \mathrm{hPa})$ over the South China Sea are close to zero, unfavorable for evening 


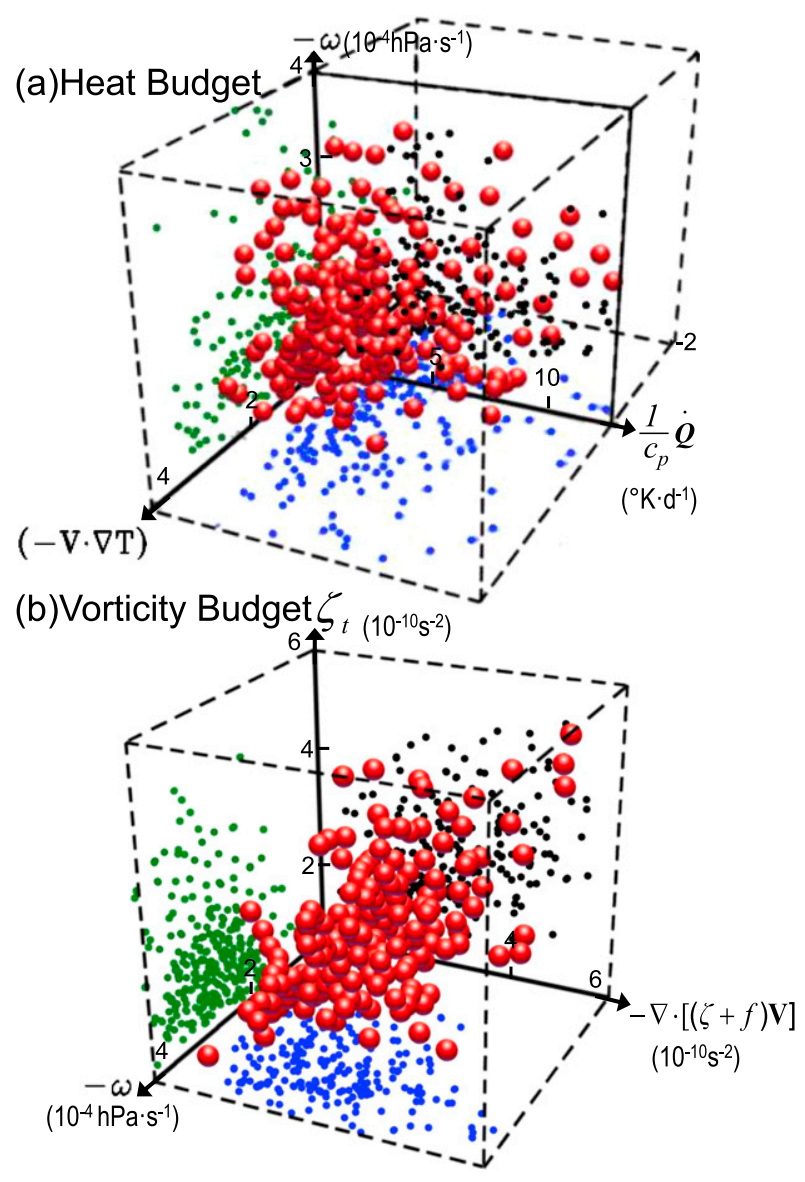

FIG. 17. Three-dimensional scatter diagrams. (a) The heat budget at $700 \mathrm{hPa}$ : upward vertical motion $(-\omega \geq 0)$ around the genesis location of rainstorms is generated/maintained by the heat advection $-\mathbf{V} \cdot \nabla T$ and diabatic heating $\left[\left(1 / c_{p}\right) \dot{Q}\right]$. (b) The vorticity budget at $700 \mathrm{hPa}$ : convergence of total vorticity flux $-\nabla \cdot[(\zeta+f) \mathbf{V}]$ induced by upward motion $(-\omega>0)$ in the vicinity of rainstorm genesis results in cyclonic spinup $\left(\zeta_{t}>0\right)$ of the environmental flow which facilitates genesis. The two-dimensional scatter diagrams are formed by two variables; the smaller dots of different colors (green, blue, and black) are projections of the three-dimensional scatters (large red balls) on the two-dimensional walls along one of the three directions on the Cartesian coordinate.

rainstorm genesis. In contrast, the reverse conditions occur at 2100 UTC. The streamfunction budget for the diurnal mode over the concerned region demonstrates the impact of the vortex-stretching process on the genesis activity of rainstorms through the diurnal mode of the regional atmospheric circulation.

\section{b. Diurnal variation of dynamic instability}

The clockwise rotation of the continent-scale divergent circulation (Figs. 18, 19) in response to the east-west continent-ocean thermal contrast and the north-south thermal differential heating can drive the clockwise rotation of the continental monsoon circulation primarily through vortex stretching (Fig. 20). Can the diurnal variation of the continental divergent and rotational circulation lead to the diurnal variation of the Charney-Stern instability criterion and result in the preferred timing of the rainstorm genesis over northern Indochina in the evening and over the South China Sea in the morning?

This question may be answered by the latitudeheight cross section of $\left(q_{y}, u\right)$ at $104^{\circ} \mathrm{E}$ (land) and $114^{\circ} \mathrm{E}$ (sea) for two 6-h windows (0900-1500 and 21000300 UTC) centered at 1200 UTC (1900 LST) and 0000 UTC (0700 LST), respectively, shown in the left- and right-hand columns of Fig. 21. A sign change of $q_{y}$ appears between $20^{\circ}$ and $22^{\circ} \mathrm{N}$, south of maximum westerlies in both time windows (Figs. 21a,b,e,f), while the corresponding $\bar{\theta}_{y}$ shows a maximum meridional gradient in the lower troposphere (Figs. 21d,h) underneath $q_{y}<0$ north of the latitude where $q_{y}$ changes its sign (Figs. 21c,g). The Charney-Stern instability criterion at both longitudes $\left(104^{\circ}\right.$ and $\left.114^{\circ} \mathrm{E}\right)$ is met by the environmental flow.

Diurnal change of $q_{y}$ and $\theta_{y}$ at the two longitudes between the two time windows are shown in Figs. 21c and $21 \mathrm{~d}$ and Figs. $21 \mathrm{~g}$ and $21 \mathrm{~h}$, respectively. Two salient features emerge from these two sets of figures:

1) The sign change in $\Delta q_{y}\left(104^{\circ} \mathrm{E}\right)$ close to $20^{\circ} \mathrm{N}$ (Fig. 21c) is the same as $q_{y}\left(104^{\circ} \mathrm{E}\right)$ (Fig. 21a), but the sign change in $\Delta q_{y}\left(114^{\circ} \mathrm{E}\right)$ (Fig. $21 \mathrm{~g}$ ) is opposite $q_{y}\left(114^{\circ} \mathrm{E}\right)$ (Fig. 21e).

2) The maximum (positive) value of $\Delta \theta_{y}\left(104^{\circ} \mathrm{E}\right)$ (Fig. 21d) appears underneath $\Delta q_{y}\left(104^{\circ} \mathrm{E}\right)<0$ north of $20^{\circ} \mathrm{N}$, while the minimum (negative) value of $\Delta \theta_{y}\left(114^{\circ} \mathrm{E}\right)($ Fig. $21 \mathrm{~h})$ exists underneath $\Delta q_{y}$ $\left(114^{\circ} \mathrm{E}\right)>0$ north of $20^{\circ} \mathrm{N}$.

These features indicate that rainstorm genesis is more likely to occur during the 0900-1500 UTC (evening) window at $104^{\circ} \mathrm{E}$ (land) and during the $2100-0300$ UTC (early morning) time window at $114^{\circ} \mathrm{E}$ (sea). In other words, when the land is warmer than the air in the evening, the Charney-Stern instability criterion is met easier by the environmental flow over the land. The reverse situation is applied to the sea. The impact of this land-sea thermal contrast on the timing preference of rainstorm genesis is reflected by the Charney-Stern instability. The response of the basic flow to the landsea thermal contrast should undergo a diurnal variation in both the $(u, \theta)$ fields; well depicted by the daily-mean component of the basic flow $(\bar{u}, \bar{\theta})$ added with their diurnal components $(\widehat{u}, \widehat{\theta})$. Note, the generations of the $(\widehat{u}, \widehat{\theta})$ component and the comparisons between 
(a)C1(63.0\%) and $\mathrm{C} 2(35.5 \%)$

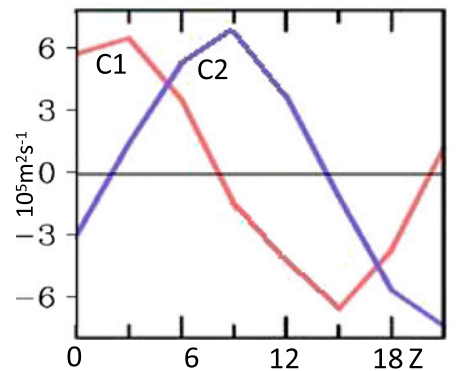

\section{(b)E1[ $\left.\widehat{\chi}_{\dot{Q}}(850 \mathrm{hPa})\right]$}

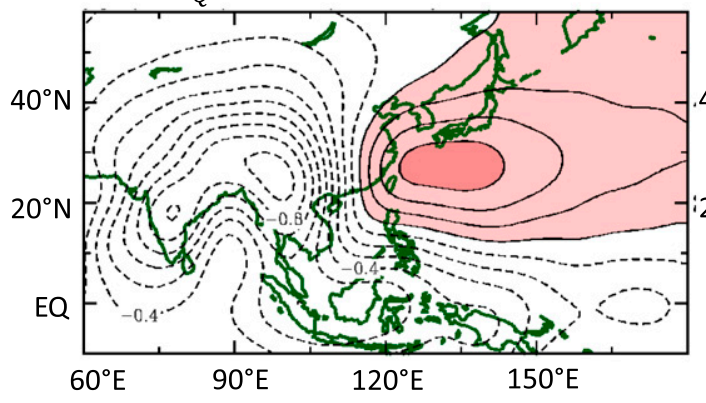

(c)E2[ $\left.\widehat{\chi}_{\dot{Q}}(850 \mathrm{hPa})\right]$

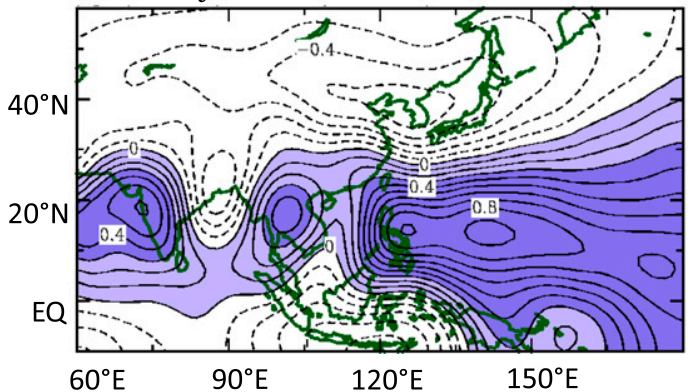

(d) $\mathrm{C} 1(63.9 \%)$ and $\mathrm{C} 2(34.6 \%)$

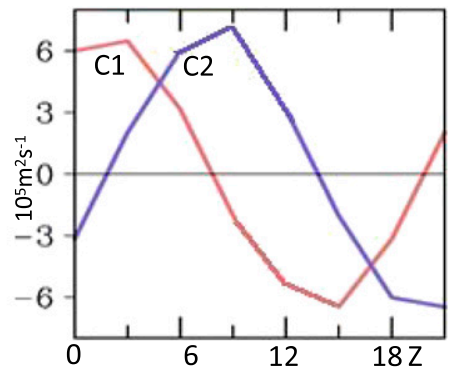

(e)E1[ $\bar{\chi}(850 \mathrm{hPa})]$

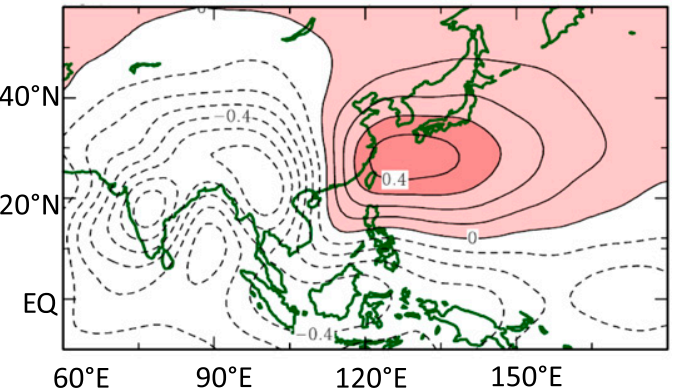

(f)E2[ $\widehat{\chi}(850 \mathrm{hPa})]$

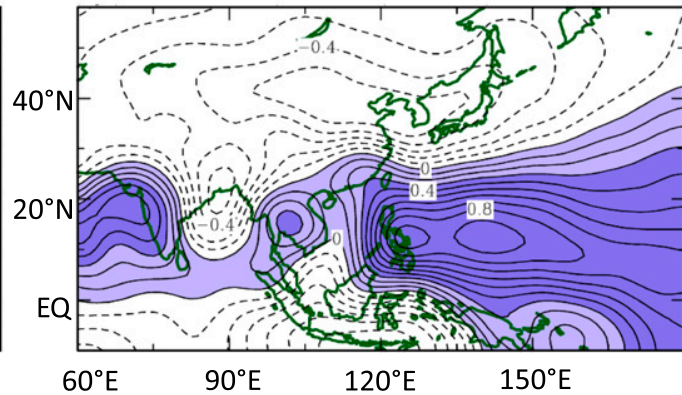

FIG. 18. Empirical orthogonal function (EOF) analysis of $\hat{\chi}_{\dot{Q}}(850 \mathrm{hPa})$ and $\widehat{\chi}(850 \mathrm{hPa})$ : eigenvalue time series of the first two eigenmodes (a) $\widehat{\chi}_{\dot{O}}(850 \mathrm{hPa})$ and $(\mathrm{d}) \hat{\chi}(850 \mathrm{hPa})$ are shown. The first two eigenvectors of both (b),(c) $\widehat{\chi}_{\dot{Q}}(850 \mathrm{hPa})$ and (e),(f) $\widehat{\chi}(850 \mathrm{hPa})$ are shown. Total variance explained by the two eigenmodes are represented by the percentage parenthesized behind $\mathrm{C} 1$ and $\mathrm{C} 2$, on the top of (a) and (d).

$(\widehat{u}, \widehat{\theta})$ and $(\bar{u}+\widehat{u}, \bar{\theta}+\widehat{\theta})$ are presented in the online supplemental material. The $\Delta(\partial q / \partial y)$ measured with $(\bar{u}+\widehat{u}, \bar{\theta}+\widehat{\theta})$ should resemble that measured with $(\bar{u}+\widehat{u}, \bar{\theta}+\widehat{\theta})$ at both longitudes $104^{\circ}$ and $114^{\circ} \mathrm{E}$ shown in Figs. 21c and 21g, and $\Delta \theta_{y}$ shown in Figs. 21d and 21h. The generations of $(\partial q / \partial y)(\widehat{u}, \hat{\theta})$ and $(\partial q / \partial y)$ $(\bar{u}+\widehat{u}, \bar{\theta}+\widehat{\theta})$, and the comparisons between are also presented in the online supplement.

\section{Conclusions}

During the active summer monsoon phase (midMay-late June) over the northern part of Southeast Asia and the southern part of East Asia, monsoon rain is primarily produced by rainstorms developed from midtropospheric cyclones (MTCs). It has been regarded by the East Asian meteorological community that rainstorms during the monsoon phase originate/ develop from mei-yu fronts. In fact, reviewing the JMA surface analysis charts, we find rainstorm geneses occur west of the mei-yu fronts. Even five rainstorms identified by the LSESREX and the SoWMEX/TiMREX have their geneses independent of the mei-yu fronts. To substantiate our argument, an identification scheme for rainstorm genesis with a two-step procedure was introduced: 1) rainstorm identification and 2) backtracking of the identified rainstorm to its genesis location. For the period 1979-2016, 

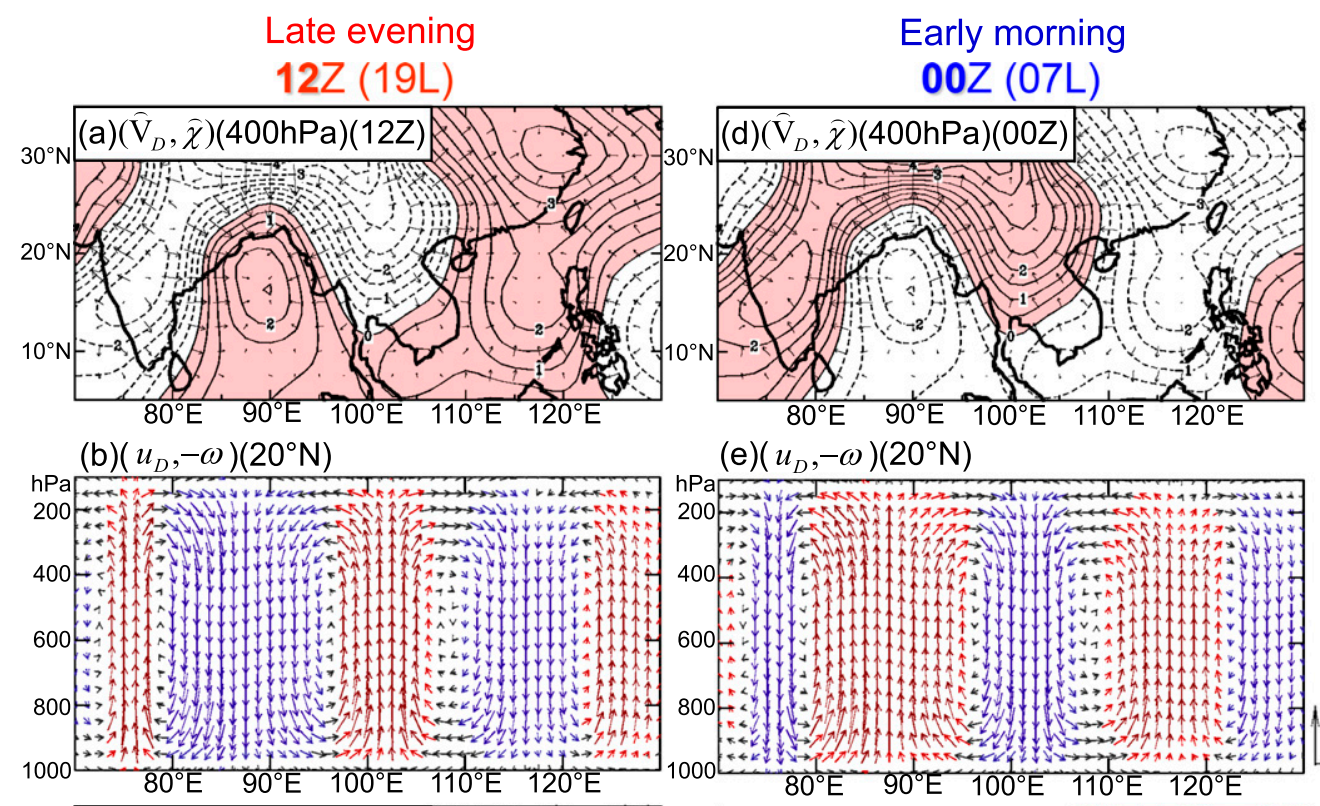

$(\mathrm{e})\left(u_{D},-\omega\right)\left(20^{\circ} \mathrm{N}\right)$

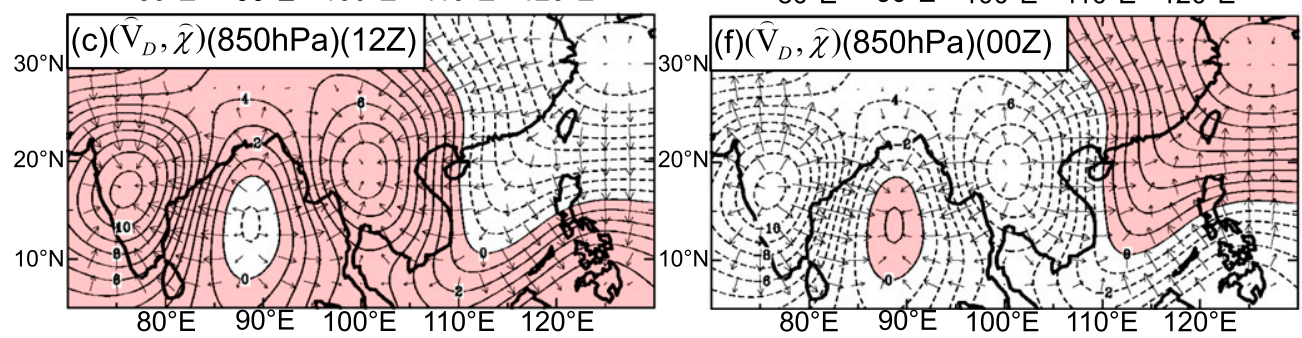

FIG. 19. Composite diurnal mode of divergent circulation $\left(\widehat{\mathbf{V}}_{D}, \widehat{\chi}\right)$ in the (a),(d) upper level (400 hPa) and (c), (f) lower level $(850 \mathrm{hPa})$ at $1200 \mathrm{UTC}(1900 \mathrm{LST})$ and $0000 \mathrm{UTC}(0700 \mathrm{LST})$, respectively. (b),(e) The corresponding east-west circulation $\left(\widehat{u}_{D},-\widehat{\omega}\right)$ at $20^{\circ} \mathrm{N}$ for both GMSs are shown.

a total of 297 rainstorms were identified and traced to their genesis locations: 248 originated over the southwestern China-northern Vietnam region and the northern South China Sea, and the other 49 rainstorm geneses occurred in the BOB-Myanmar region. Our efforts in this study focus only on the former group of rainstorms. Salient geographic features of rainstorm genesis locations in this group included the following:

1) A maximum occurrence of genesis appears in the evening over the northern Vietnam-southwestern China region, while a minor maximum exists in the morning over the northern South China Sea.

2) Geneses occur in the midtroposphere $(700-500-\mathrm{hPa}$ layer).

3) All 248 geneses are not directly linked to mei-yu fronts, in contrast with the traditional perception that rainstorms/MCSs evolve from mei-yu fronts.
These rainstorm genesis features are attributed to a special structure for the large-scale environmental flow, particularly in the midtroposphere, favorable for rainstorm genesis in Southeast/East Asia.

During May-June, the development of the Tibetan anticyclone moves the westerly jet northward across the plateau. The confluence of the midtropospheric anticyclonic flow around the eastern plateau and the lower-tropospheric monsoon southwesterlies develops a narrow trough in parallel with the monsoon southwesterlies with its western end located in southwestern China. The Charney-Stern instability criterion is met over this trough in the midtroposphere. When this environmental flow is intensified by the cold surgelike Tibetan northwesterly flow, coupled with a short-wave trough along the eastern seaboard of northeast Asia, rainstorm genesis occurs. For a great majority of these rainstorms, their geneses are triggered by the mechanism independent of East Asian fronts. 

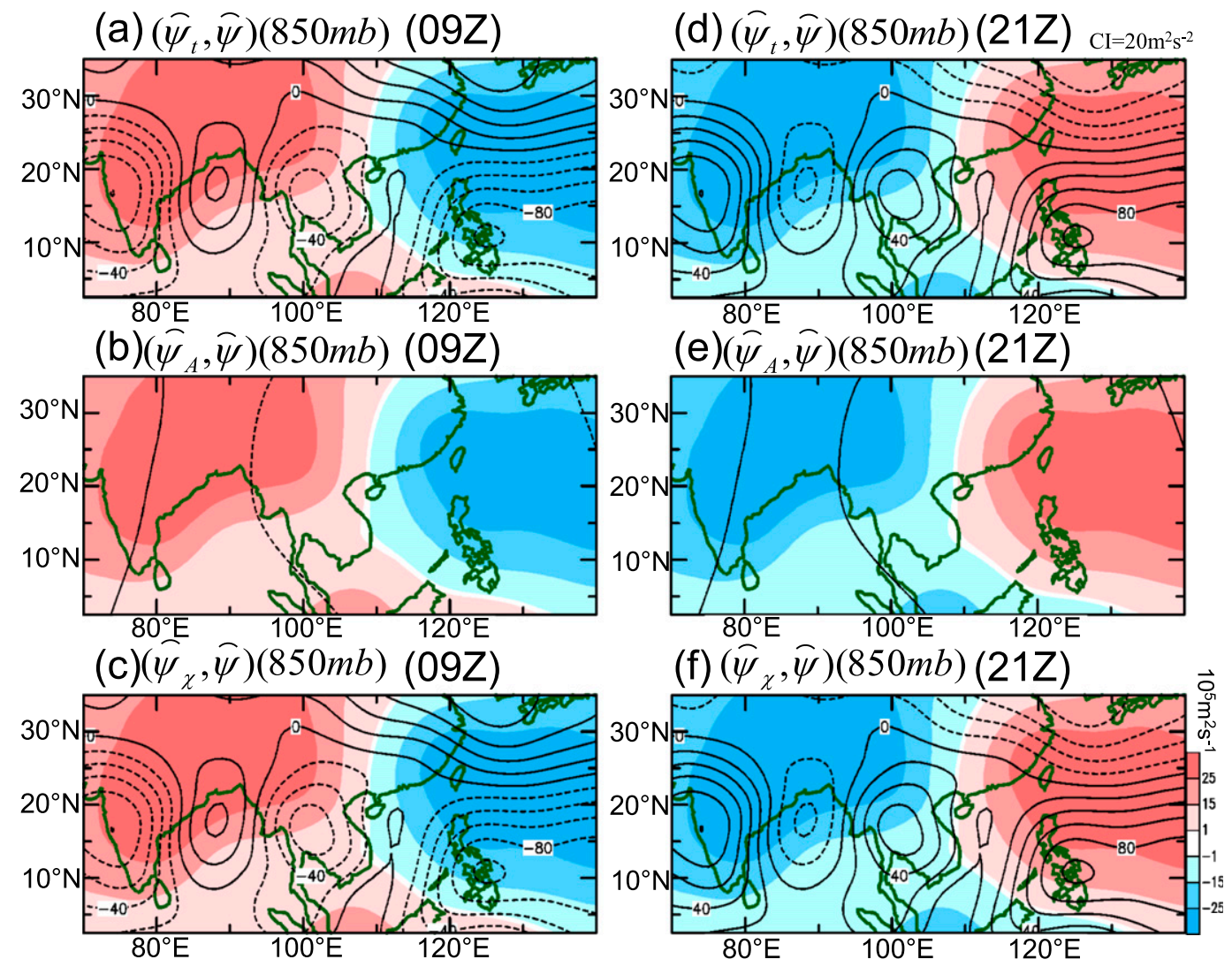

FIG. 20. Diurnal mode of the streamfunction budget at $0900 \mathrm{UTC}$ for (a) $\left(\widehat{\psi}_{t}, \widehat{\psi}\right)(850 \mathrm{hPa}),(\mathrm{b})\left(\widehat{\psi}_{A}, \widehat{\psi}\right)(850 \mathrm{hPa})$, (c) $\left(\widehat{\psi}_{\chi}, \widehat{\psi}\right)(850 \mathrm{hPa}) .(\mathrm{d})-(\mathrm{f})$ As in (a)-(c), but $2100 \mathrm{UTC}$. The contour interval of $\left(\widehat{\psi}_{t}, \widehat{\psi}_{A}, \widehat{\psi}_{\chi}\right)(850 \mathrm{hPa})$ is $20 \mathrm{~m}^{2} \mathrm{~s}^{-2}$, while the color scale of $\psi(850 \mathrm{hPa})$ is shown at the bottom right of (f).

The diurnal variation of the regional atmospheric circulation in Asia and its surrounding oceans in response to the land-sea contrast and north-south differential heating is a clockwise rotation of the regional divergent circulation, as observed by Chen (2006). Accompanying the diurnal clockwise rotation of this divergent circulation, a diurnal oscillation of the east-west circulation appears between the land (northern Indochina) and the sea (South China Sea). The diurnal oscillation of upward vertical motions between these two regions results in the diurnal oscillation of the vorticity tendency, primarily through vortex stretching. In turn, this dynamic process spins up the atmospheric flow over land in late evening and over the sea in early morning, and results in the corresponding diurnal variation of rainstorm genesis between land and sea, as observed by this study.

New findings of the monsoon rainstorm genesis activity during May and June in this study offer suggestions for several future research tasks:
1) The development of a rainstorm from its genesis is accompanied by a downward penetration of vorticity anomaly. Analyzing the vertical structure of mesoscale convective vortices (MCVs) observed by the Bow Echo and Mesoscale Convective Vortex Experiment (BAMEX; Davis et al. 2004), Davis and Trier (2007) also found the downward development of MCV. Simulating some BAMEX MCVs with the Advanced Research Weather Research and Forecasting Model (Skamarock et al. 2005), Davis and Galarneau (2009) realized the vertical development of MCV vorticity anomaly includes both the downward penetration and the generation from the planetary boundary layer. The rainstorm and MCV may differ in some basic characteristics, but the BAMEX effort suggests some direction for our mechanism search for the down penetration of rainstorms.

2) Revealed from the longitude-time diagrams of rainfall and vorticity, the eastward propagation of the five rainstorms during May and June 2008 are 

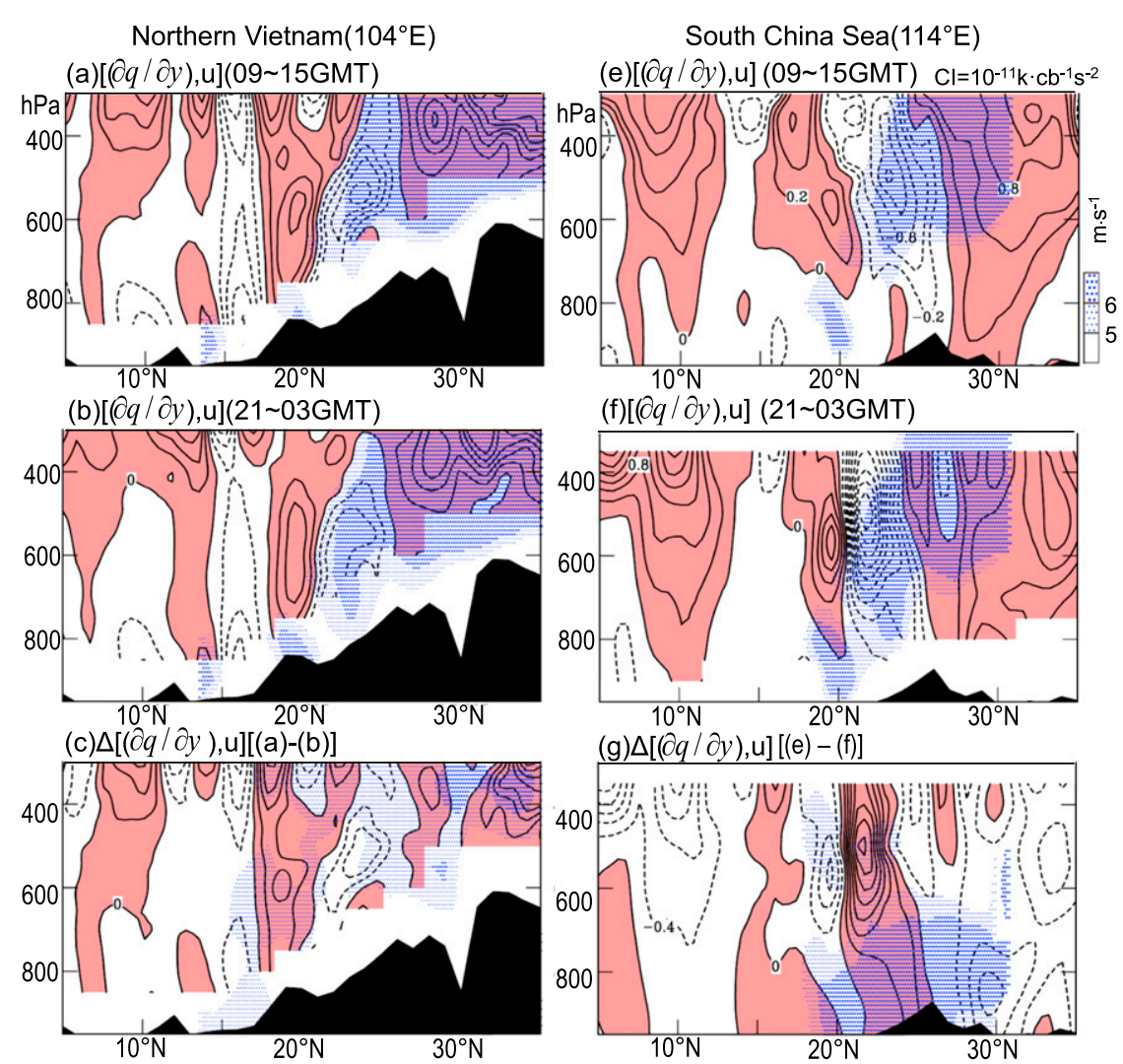

(d) $\Delta \theta_{y}[=\theta(09-15 Z)-\theta(21-03 Z)]$
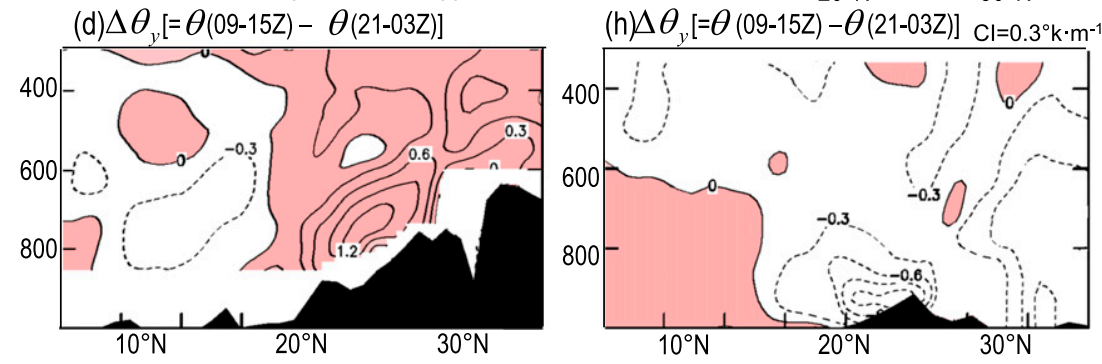

FIG. 21. Diurnal change of $(\partial q / \partial y, u)$ and $\theta$ at (left) $104^{\circ} \mathrm{E}$ and (right) $114^{\circ} \mathrm{E}$ : (a), (e) for 0900 1500 UTC; (b),(f) for 2100-0300 UTC; (c) difference between (a) and (b); (g) difference between (e) and (f); (d) $\Delta \theta\left[=\theta\left(0900-1500\right.\right.$ UTC) $-\theta(2100-0300$ UTC) $]$ at $104^{\circ} \mathrm{E}$; and (h) as in (d), but at $114^{\circ} \mathrm{E}$. The contour interval of all concerned variables in (a)-(c) and (e)-(g) are shown on the top right of (e), while that for (d) and (h) is shown on the top right of (h).

modulated by an intraseasonal mode. Juxtaposed with the South China Sea monsoon trough to the west and the western subtropical Pacific anticyclone to the east, the rainfall produced by these rainstorms should be affected by the modulation of these two synoptic systems by this intraseasonal mode. A future effort should be made to examine the impact of this modulation on the rainfall production of rainstorms over East Asia.

3) The geneses of BOB-Myanmar rainstorms occur ahead of the midtropospheric BOB trough. First, this synoptic system is different from that for the southwestern China-northern Vietnam rainstorm. Second, the distance between the BOB-Myanmar region and East Asia is $\geq 2000 \mathrm{~km}$. For the BOB-Myanmar rainstorms to propagate through such a long distance, it is likely these rainstorms should be propagated eastward by different weather systems across South and Southeast Asia. The genesis and eastward-propagation mechanisms for these rainstorms are likely different from the southwestern China-northern Vietnam rainstorms and need future examination.

Acknowledgments. The effort for this research project was supported partially by the Cheney Research fund and NSF Grant ATM-0136220. M.-C. Yen's participation of 
this project is supported by Grant MOST106-2111-M-008005. Comments and suggestions made by three anonymous reviewers were helpful for us to improve this paper. We would also like to thank Amanda Black for her help finalizing this paper.

\section{REFERENCES}

Ashouri, H., K.-L. Hsu, S. Sorooshian, D. K. Braithwaite, K. R. Knapp, L. D. Cecil, B. R. Nelson, and O. P. Prat, 2015: PERSIANN-CDR: Daily precipitation climate data record from multisatellite observations for hydrological and climate studies. Bull. Amer. Meteor. Soc., 96, 69-83, doi:10.1175/ BAMS-D-13-00068.1.

Atlas, R., and Coauthors, 2001: The effects of marine winds from scatterometer data on weather analysis and forecasting. Bull. Amer. Meteor. Soc., 82, 1965-1990, https://doi.org/10.1175/ 1520-0477(2001)082<1965:TEOMWF $>2.3$.CO;2.

Blersch, D. J., and T. C. Probert, 1991: Geostationary meteorologica satellite systems-An overview. J. Pract. Appl. Space, 2, 1-13.

Burpee, R., 1972: The origin and structure of easterly waves in the lower troposphere of North Africa. J. Atmos. Sci., 29, 77-90, https://doi. org/10.1175/1520-0469(1972)029<0077:TOASOE > 2.0.CO;2.

Chang, J.-H., 1972: Atmospheric Circulation Systems and Climates. Oriental Publishing Co., 328 pp.

Charney, J., and M. Stern, 1962: On the stability of internal baroclinic jets in a rotating atmosphere. J. Atmos. Sci., 19, 159-172, https:// doi.org/10.1175/1520-0469(1962)019<0159:OTSOIB > 2.0.CO;2.

Chen, S. J., Y. H. Kuo, W. Wang, Z. Y. Tao, and B. Cui, 1998: A modeling case study of heavy rainstorms along the Mei-Yu front. Mon. Wea. Rev., 126, 2330-2351, https://doi.org/10.1175/ 1520-0493(1998)126<2330:AMCSOH > 2.0.CO;2.

Chen, T. C., 2006: Variation of the Asian monsoon water vapor budget: Interaction with the global-scale modes. The Asian Monsoon, B. Wang, Ed., Springer Praxis Books Series, Springer, 417-458.

- , and W. E. Baker, 1986: Global diabatic heating during FGGE SOP-1 and SOP-2. Mon. Wea. Rev., 114, 2578-2589, https:// doi.org/10.1175/1520-0493(1986)114<2578:GDHDFS>2.0.CO;2.

heating associated with the Madden-Julian oscillation J. Geophys. Res., 96, 13163-13177, https://doi.org/ 10.1029/91JD01356.

— , and $ـ$, 1991b: Interaction between intraseasonal oscillations of the midlatitude flow and tropical convection during 1979 northern summer: The Pacific Ocean. J. Climate, 4, 653-671, https://doi.org/10.1175/1520-0442(1991)004<0653: IBIOOT $>2.0 . \mathrm{CO} ; 2$

—, and J. M. Chen, 1997: On the relationship between the streamfunction and velocity potential of the Madden-Julian oscillation. J. Atmos. Sci., 54, 679-685, https://doi.org/10.1175/ 1520-0469(1997)054<0679:OTRBTS > 2.0.CO;2.

- , S.-Y. Wang, W.-R. Huang, and M.-C. Yen, 2004: Variation of the East Asian summer monsoon rainfall. J. Climate, 17, 744-762, https://doi.org/10.1175/1520-0442(2004)017<0744: VOTEAS $>2.0 . \mathrm{CO} ; 2$.

_- W.-R. Huang, and M.-C. Yen, 2011: Interannual variation of the late spring-early summer monsoon rainfall in the northern part of the South China Sea. J. Climate, 24, 4295-4313, https:// doi.org/10.1175/2011JCLI3930.1.

Clark, J. D., 1983: The GOES user's guide. NASA Tech. Rep. C 55.202:983, U.S. Department of Commerce, 165 pp.
Davis, C. A., and S. B. Trier, 2007: Mesoscale convective vortices observed during BAMEX. Part I: Kinematic and thermodynamic structure. Mon. Wea. Rev., 135, 2029-2049, https://doi.org/ 10.1175/MWR3398.1.

, and T. J. Galarneau Jr., 2009: The vertical structure of mesoscale convective vortices. J. Atmos. Sci., 66, 686-704, https:// doi.org/10.1175/2008JAS2819.1.

— , and W.-C. Lee, 2012: Mesoscale analysis of heavy rainfall episodes from SoWMEX/TiMREX. J. Atmos. Sci., 69, 521-537, https://doi.org/10.1175/JAS-D-11-0120.1.

— and Coauthors, 2004: The Bow Echo and MCV Experiment: Observations and opportunities. Bull. Amer. Meteor. Soc., 85, 1075-1093, https://doi.org/10.1175/BAMS-85-8-1075.

Faller, K., 2005: MTSAT-1R: A multifunctional satellite for Japan and the Asia-Pacific Region. Proc. 56th IAC 2005, Fukuoda, Japan, International Astronautical Congress, IACIAC-05B3.2.04.

Haynes, P. H., and M. E. McIntyre, 1987: On the evolution of vorticity and potential vorticity in the presence of diabatic heating and frictional or other forces. J. Atmos. Sci., 44, 828-841, https://doi.org/10.1175/1520-0469(1987)044<0828: OTEOVA $>2.0 . \mathrm{CO} ; 2$.

Holton, J. R., and G. J. Hakim, 2013: An Introduction to Dynamic Meteorology. 5th ed. Academic Press, 535 pp.

Huffman, G. J., and D. T. Bolvin, 2013: GPCP version 2.2 SG combined precipitation data set documentation. NASA GSFC Doc., 46 pp., ftp://meso.gsfc.nasa.gov/pub/gpcp-v2.2/doc/ V2.2_doc.pdf.

—, and - 2015: Real-time TRMM Multi-Satellite Precipitation Analysis data set documentation. NASA TRMM Doc., 47 pp., http://pmm.nasa.gov/sites/default/files/document_files/ 3B4XRT_doc_V7.pdf.

Jou, B. J.-D., W. C. Lee, and R. H. Johnson, 2011: An overview of SoWMEX/TiMREX. The Global Monsoon System: Research and Forecast, 2nd ed. C. P. Chang et al., Eds., World Scientific Publishing Co., 303-318.

Lai, H.-W., C. A. Davis, and B. J.-D. Jou, 2011: A subtropical oceanic mesoscale convective vortex observed during SoWMEX/TiMREX. Mon. Wea. Rev., 139, 2367-2385, https://doi. org/10.1175/2010MWR3411.1.

Lau, K.-M., and M.-T. Li, 1984: The monsoon of East Asia and its global associations-A survey. Bull. Amer. Meteor. Soc., 65, 114-125, https://doi.org/10.1175/1520-0477(1984)065<0114: TMOEAA $>2.0 . \mathrm{CO} ; 2$.

_, and S. Yang, 1997: Climatology and interannual variability of the Southeast Asian summer monsoon. Adv. Atmos. Sci., 14, 141-162, https://doi.org/10.1007/s00376-997-0016-y.

Lau, W. K.-M., 1997: South China Sea Monsoon Experiment observed from satellites. Eos, Trans. Amer. Geophys. Union, 78, 599-603, https://doi.org/10.1029/97EO00361.

Liebmann, B., and C. A. Smith, 1996: Description of a complete (interpolated) outgoing longwave radiation dataset. Bull. Amer. Meteor. Soc., 77, 1275-1277.

Meteorological Services Centre Japan, 1997: GMS-5 User's Guide. 3rd ed. Meteorological Satellite Center of Japan, $190 \mathrm{pp}$.

NCEP, 2003: The GFS atmospheric model. NCEP Office Note 442, 14 pp., http://nws.noaa.gov/ost/climate/STIP/ AGFS_DOC_1103.pdf.

, 2016: The Global Forecast System (GFS)—Global Spectral Model (GSM) (GSM version 13.0.2). NOAA/NWS, accessed 19 June 2016, http://www.emc.ncep.noaa.gov/GFS/doc.php.

Ninomiya, K., and T. Murakami, 1987: The early summer rainy season (Baiu) over Japan. Monsoon Meteorology, C.-P. Chang 
and T. N. Krishnamurti, Eds., Oxford University Press, 93-121.

Ramage, C. S., 1952: Variation of rainfall over south China through the wet season. Bull. Amer. Meteor. Soc., 33, 308-311.

Rienecker, M. M., and Coauthors, 2008: The GEOS-5 Data Assimilation System-Documentation of versions 5.0.1, 5.1.0, and 5.2.0. NASA GSFC Tech. Rep. NASA/TM-2008-104606, Vol. 27, 101 pp., https://citeseerx.ist.psu.edu/viewdoc/download? doi=10.1.1.496.8365\&rep=rep1\&type=pdf.

Saha, S., and Coauthors, 2010: The NCEP Climate Forecast System Reanalysis. Bull. Amer. Meteor. Soc., 91, 1015-1058, https:// doi.org/10.1175/2010BAMS3001.1.

Sanders, F., 1984: Quasi-geostrophic diagnosis of the monsoon depression of 5-8 July 1979. J. Atmos. Sci., 41, 538-552, https://doi.org/ 10.1175/1520-0469(1984)041<0538:QGDOTM >2.0.CO;2.

Skamarock, W. C., J. B. Klemp, J. Dudhia, D. O. Gill, D. M. Barker, W. Wang, and J. G. Powers, 2005: A description of the Advanced Research WRF version 2. NCAR Tech. Note NCAR/TN468+STR, 88 pp., https://doi.org/10.5065/D6DZ069T.

Taiwan EAMEX Committee, 2007: Science Plan-East Asian Monsoon Field Experiment (EAMEX): Participation of the MAHASRI (post-GAME) International Field Experiment.
Iowa State University, Ames, Iowa, and National Central University, Chung-Li, Taiwan, 49 pp., http://www.public.iastate.edu/ $\sim$ tmchen/Download/EAMEX_proposal. English.pdf.

Tao, S., and Y. Ding, 1981: Observational evidence of the influence of the Qinghai-Xizang (Tibet) plateau on the occurrence of heavy rain and severe convective storms in China. Bull. Amer. Meteor. Soc., 62, 23-30, https://doi.org/10.1175/1520-0477 (1981)062<0023:OEOTIO>2.0.CO;2.

Thorncroft, C. D., and B. J. Hoskins, 1994: An idealized study of African easterly waves. I: A linear view. Quart. J. Roy. Meteor. Soc., 120, 953-982, https://doi.org/10.1002/ qj.49712051809.

Wang, C.-C., J. C.-S. Hsu, G. T.-J. Chen, and D.-I. Lee, 2014: A study of two propagating heavy-rainfall episodes near Taiwan during SoWMEX/TiMREX IOP-8 in June 2008. Part I: Synoptic evolution, episode propagation, and model control simulation. Mon. Wea. Rev., 142, 2619-2643, https://doi.org/ 10.1175/MWR-D-13-00331.1.

Wang, S.-Y., and T.-C. Chen, 2008: Measuring East Asian summer monsoon rainfall contributions by different weather systems over Taiwan. J. Appl. Meteor. Climatol., 47, 2068-2080, https:// doi.org/10.1175/2007JAMC1821.1. 